\title{
Modeling Sun Glitter at L-Band for Sea Surface Salinity Remote Sensing With SMOS
}

\author{
Nicolas Reul, Joseph Tenerelli, Bertrand Chapron, and Philippe Waldteufel
}

\begin{abstract}
Since the sun is an extremely strong radiation source at L-band, accounting for sun glint over the ocean, i.e., solar radiation reflected by the sea surface toward downward-looking radiometers, raises a significant challenge for the remote sensing of sea surface salinity. This paper describes a dedicated physical model for sun glint at L-band frequencies and provides quantitative and qualitative estimates of the sun glint contamination impinging the antenna of the Microwave Imaging Radiometer with Aperture Synthesis interferometer onboard the future European Space Agency's Soil Moisture and Ocean Salinity (SMOS) mission. The sun brightness temperature expected during the mission period is first estimated from past solar flux data with an expected range of $1.1 \times 10^{5} \mathrm{~K}$ to about $9 \times 10^{5} \mathrm{~K}$. Numerical simulations of the predicted illumination of the SMOS antenna by solar radiation scattered by the rough sea surface are then performed at key dates of the seasonal cycle using different asymptotic scattering models and several representative surface conditions. Although the center of the sun's glitter pattern will never be located within the useful part of SMOS' synthesized field of view, the expected contamination due to roughness scattering will range between $0 \mathrm{~K}$ and about $500 \mathrm{~K}$, depending on the target position, the season period, the roughness state at the target, and the level of solar activity at the time of measurements. In particular, we find the sun glint contamination to be more intense when SMOS will probe ocean surfaces in the Southern Hemisphere, reaching maxima in descending passes with highest values expected at dates around winter solstices.
\end{abstract}

Index Terms-Microwave radiometry, ocean remote sensing, rough surface scattering, sea surface salinity (SSS), sun glitter.

\section{INTRODUCTION}

$\mathbf{S}$ EA SURFACE salinity (SSS) is a crucial variable needed to understand ocean dynamics and its role in the water cycle and climate system. New developments in L-band radiometry will soon provide the key technology to obtain global surface salinity estimates for the first time. Indeed, two new satellite missions are now being developed to map SSS: the European Space Agency's Soil Moisture and Ocean Salinity (SMOS) mission and the proposed U.S./Argentinean Aquarius/SAC-D mission. The goal is to achieve a root-mean-square uncertainty on the retrieved SSS of about 0.1 psu on monthly time scales over the global ocean.

Manuscript received June 2, 2006; revised October 11, 2006. This work was supported by the European Space Agency under Contract 16027/02/NL/GS.

N. Reul, J. Tenerelli, and B. Chapron are with the Laboratoire d'Océanographie Spatiale, Institut Français de Recherche et d'Exploitation de la Mer, 29200 Plouzané, France (e-mail: nreul@ifremer.fr).

P. Waldteufel is with the Institut Pierre et Simon Laplace, Service d'Aéronomie, Centre National de la Recherche Scientifique, 91371 Paris, France.

Color versions of one or more of the figures in this paper are available online at http://ieeexplore.ieee.org.

Digital Object Identifier 10.1109/TGRS.2006.890421
A detailed analysis of various geophysical error sources that influence the remote sensing of ocean surface salinity using spaceborne L-band radiometers has been performed by Yueh et al. [1]. The main geophysical sources of error that were identified are listed as follows:

- sea surface temperature;

- atmosphere dry air and water vapor;

- clouds;

- sea surface roughness;

- ionospheric effects;

- solar system and galactic radiation.

Beyond these sources, Yueh et al. [1] found that solar radiation poses a significant challenge for the remote sensing of ocean surface salinity. The sun is indeed an extremely strong radiation source at the L-band, exhibiting a time-dependent blackbody temperature that ranges between $100000 \mathrm{~K}$ and several million kelvins, depending on the solar activity. Then, two distinct mechanisms may contribute to the solar radiation intercepted by a radiometer antenna: one is the reflection of sun radiation by the Earth surface (sun glitter effects), and the other is the direct leakage into the antenna.

Direct and indirect sun effects in 2-D Aperture Synthesis Radiometry Imaging with SMOS have been studied, and cancellation techniques to remove these effects during the image reconstruction process have been discussed and proposed in [2]-[4]. Due to aliasing in the Fourier synthesis imaging, replicas of the direct sun images appear in the useful part of the instrument field of view (FOV) overlapped with the image of the Earth, corrupting it and making it useless for scientific purposes. The direct sun indeed appears in $96.81 \%$ of the brightness temperature images: half in front of the array, half behind, and only in the regions corresponding to the sky. Assuming a perfectly smooth Earth surface (i.e., no roughness), the reflected sun appears in Earth regions that are outside the useful FOV. Accordingly, this should not be a problem, except for the amplitude of the response that may spill in the FOV, because of Gibbs phenomenon. These effects affect the average brightness temperature of the Earth, which translates into a reconstructed image bias. To remove these biases, visibility samples that would be measured by the instrument corresponding to unitary point sources located at the positions of the direct or reflected sun can be computed and subtracted from the measured visibilities prior to the reconstruction process. The visibilities computed to evaluate direct sun impact depend not only on the direct sun alias position that is known a priori (since it depends on the relative position and orientation between the sun and the platform, as well as its attitude) but 
also on the brightness temperature of the sun. The latter one depends on the solar cycle and can exhibit strong fluctuations with time. A technique was derived in [2] to estimate the sun brightness temperature from the SMOS observables themselves, which effectively reduces the impact of the direct sun image. While the shape of the direct sun and moon images are not perturbed, the reflected ones will be distorted by the rough Earth surface. To evaluate the corresponding sun glint effects on visibilities, a forward model that predicts the sun glint intensity patterns impinging the SMOS antenna is therefore required in the SMOS Level-1 image reconstruction processing. In Level-2 SSS retrieval processing from multiangular reconstructed brightness temperature data, a forward model would be also required to define flags to determine data potentially corrupted by sun glint in the multiangular L1C brightness temperature data sets.

This paper therefore focuses on evaluating the signatures associated with solar radiation scattered by the rough sea surface toward SMOS' Microwave Imaging Radiometer with Aperture Synthesis (MIRAS) antenna. Hereafter, we refer to this contribution as the sun glitter contamination. Experimental evidence of the strong impact of sun glitter on the passive microwave sensing of the ocean using L-band radiometers was first given in 1974 by Swift [5], who analyzed the forward scattering of sun microwave radiation from the Cape Cod Canal in Massachusetts. Data were collected at 1.4, 4.0, and 7.5 GHz for the horizontal polarization (H-polarization) and the vertical polarization (V-polarization) at a fixed nadir viewing angle of $40^{\circ}$. As the sun passed through the antenna beam, Swift found that the excess temperature due to reflected solar radiation increased dramatically with decreasing frequency and was polarization dependent. The sun was found to be such a dominating source at $1.4 \mathrm{GHz}$ that the horizontally polarized component saturated the radiometer. As shown by Wentz [6], these sun-glitter effects might be estimated using an approximate scattering model to compute the forward scattering of the sun radiation from the rough water surface. In this paper, we provide qualitative and quantitative estimates of the expected sun glint pollution impinging the MIRAS sensor during the SMOS mission period (2007-2010).

Key features of the solar radiation at $1.4 \mathrm{GHz}$ are first reviewed. Using available ground-based measurements of the past solar radiation fluxes at L-band frequencies and the reported signals' 11-year periodicity, a potential time series of the sun brightness temperature is estimated at $1.4 \mathrm{GHz}$ for the future SMOS mission period.

In the second part, we present the theoretical formulation of the sun glint forward modeling problem at surface level. We then describe the model for the rough sea surface bistatic scattering coefficients at L-band. In order to test the sensitivity of the results to the model, two differing asymptotic scattering theories are used, namely: 1) the Small-Slope Approximation (SSA) theory [7], [8] and 2) the Kirchhoff Approximation (KA) [9].

In the third part, the impact of the indirect leakage by sun glint on simulated rough sea surfaces is estimated for two selected characteristic days of the sun seasonal cycle (winter solstice and spring equinox).
To assess the magnitude of the sun glint contamination within the antenna FOV during the sensor propagation for different roughness states, we first assumed uniform (constant) surface wind fields, conducting calculations for the global ocean average wind speed condition $(7 \mathrm{~m} / \mathrm{s}$ ) and for two extremes (lowwind and high-wind conditions), with every other geophysical surface field being kept constant. Only isotropic rough sea surfaces were considered in these first series of simulations.

The impact of roughness anisotropy is further estimated for uniform wind speeds but with varying directions derived from representative QuikSCAT products.

We show that sun glint contamination within the right-hand part of the MIRAS' useful FOV might occur with an intensity ranging between $0 \mathrm{~K}$ and about $500 \mathrm{~K}$, depending on the target position, the season period, the roughness state at the target, and the level of solar activity at the time of measurements. In particular, we find the sun glint contamination to be more intense when SMOS probes ocean surfaces in the Southern Hemisphere, reaching the maxima in descending passes, with the highest values expected at dates around winter solstices.

In the last section, we finally discuss potential errors in the sun glint modeling, validation strategies, methods of correction, and flagging techniques as currently foreseen in the SMOS salinity data processing.

\section{BRIGHTNESS TEMPERATURE OF THE SUN AT $21 \mathrm{~cm}$ DURING SMOS MISSION}

\section{A. Solar Radio Emission at $1.4 \mathrm{GHz}$}

Radio emission from the sun arises from several different phenomena. It can be divided into three main components on the basis of characteristic time scales of intensity variation, i.e.,

$$
T_{\mathrm{sun}}(t)=T_{R}(t)+T_{S}(t)+T_{\mathrm{QS}}
$$

where the following conditions hold.

1) $T_{R}$ is the rapidly varying $R$-component, consisting of the second and minute-duration bursts produced by the active sun components: sunspots (manifestations of magnetically disturbed conditions at the sun's visible surface), flares (huge explosions on the surface of the sun), and other transient activity.

2) $T_{S}$ is the slowly varying $S$-component, comprising all slower variations, over hours to decades.

3) $T_{\mathrm{QS}}$ is the quiet sun component, i.e., a constant base level corresponding to an extrapolated zero of activity.

A comprehensive discussion of the issues pertaining to the 1.4-GHz solar radio activity can be found in [10]. At such a frequency (wavelength of $21 \mathrm{~cm}$ ), the quiet sun component $T_{\mathrm{QS}}$ is seen as a blackbody of about $100000 \mathrm{~K}$. The other two components in (1) are related to the sunspot activity over the sun disk and can be locally as high as 2 million $\mathrm{K}$ at L-band.

As discussed in [10], although the degree of polarization of sun emission at L-band varies in amount and sense within the active region and over the solar disc, integrating the emission over the entire disc probably leads to an observed polarization 
averaging out. Since we consider this integrated signal as the source for evaluating the sun glint contribution at $1.4 \mathrm{GHz}$, we assume in what follows unpolarized downwelling sun radiation.

\section{B. Radio Fluxes}

The sun radio fluxes are typically expressed in solar flux units (sfu) $\left(1 \mathrm{sfu}=10^{-22} \mathrm{~W} \cdot \mathrm{m}^{-2} \cdot \mathrm{Hz}^{-1}\right)$. These fluxes, which are hereafter denoted $F_{\lambda}$ at wavelength $\lambda$, are defined as the brightness integrated over the entire solar disc, i.e.,

$$
F_{\lambda}=B_{\lambda} \Omega_{\text {sun }}
$$

where the integrated brightness $B_{\lambda}$ is expressed in watts per square meter per hertz per steradian, and $\Omega_{\text {sun }}$ (steradian) is the solid angle intercepting the sun as seen from the Earth, i.e.,

$$
\Omega_{\mathrm{sun}}=2 \pi\left[1-\cos \left(\beta_{\mathrm{sun}} / 2\right)\right]=8.2 \times 10^{-5} \mathrm{sr}
$$

where $\beta_{\text {sun }} / 2$ is the angular radius of the sun as viewed from the Earth. At $1.4 \mathrm{GHz}, \beta_{\text {sun }} / 2 \simeq 0.293^{\circ}$, which is $10 \%$ larger than the optical angular radius [11].

Daily values of solar fluxes at various wavelengths are available from continuous ground-based routine measurements around the world. There are solar flux measurements available at $1.415 \mathrm{GHz}$ made by the U.S. Air Force, Sagamore Hill, MA, since 1966. They can be obtained through the National Geophysical Data Center, Boulder, CO. These data sets also include other solar flux measurements that has been conducted at $1415 \mathrm{MHz}$ since 1988 from radiometers in Palehua, HI; San Vito, Italy; and Learmonth, W.A., Australia; however, the data have not been quality controlled. Solar flux data at $1 \mathrm{GHz}$ have also been collected daily at the Nobeyama Radio Observatory, Nagano, Japan, since 1957.

\section{Sun Brightness at 1.4 GHz During SMOS Mission}

The integrated sun brightness temperature at wavelength $\lambda$ can be deduced from $F_{\lambda}$ measurements using

$$
T_{\text {sun }}(t)=\frac{\lambda^{2} F_{\lambda}(t)}{2 k \Omega_{\text {sun }}}
$$

where $k=1.3810^{-23} \mathrm{~J} \cdot \mathrm{K}^{-1}$ is Boltzmann's constant.

The SMOS mission nominal lifetime span (2007-2010) should approximately correspond to the following previous periods of the so-called 11-year solar cycle: 1996-1999, $1985-1988,1974-1977, \ldots$. The last full solar cycle is (1986.8-1996.4), and a cycle starts from an activity minimum [12]. Therefore, the SMOS mission (whose scheduled launch date is near the end of 2007) should start several months after a minimum solar activity, with a continuously increasing activity during the mission lifetime. In recent cycles, the solar maxima has followed the solar minima by just four years so that a solar maximum is expected in 2010 .

In Fig. 1, we plot the daily sun brightness temperature at $1.4 \mathrm{GHz}$ deduced from $F_{21}$ measurements conducted at Sagamore Hill, Learmonth, and Palehua during the period

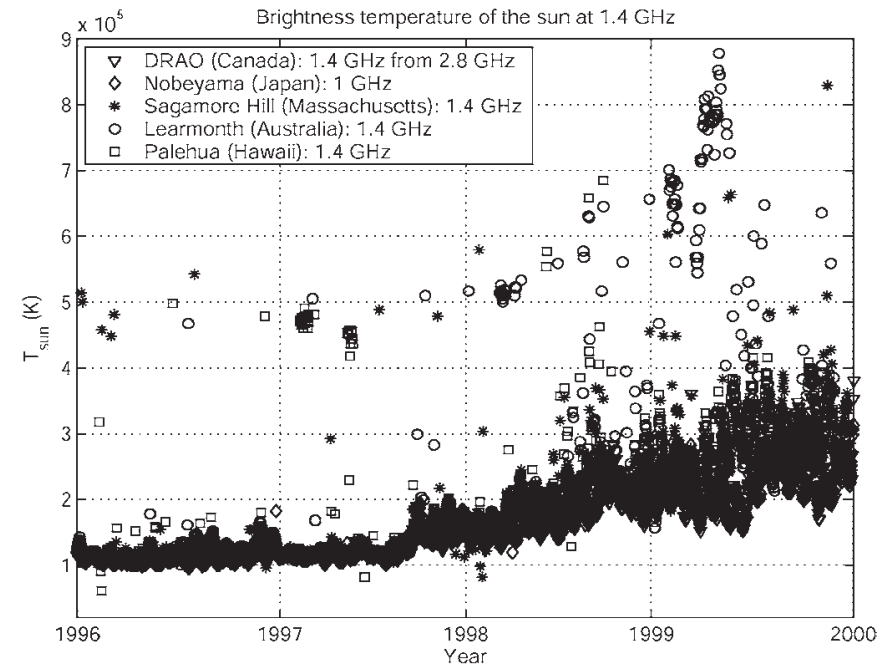

Fig. 1. Daily sun brightness temperature at $1.4 \mathrm{GHz}$ from 1996 to 2000 deduced from several measurements of the solar flux integrated over the sun disc.

1996-2000. We have also plotted the 1-GHz brightness temperature data deduced from Nobeyama measurements and the brightness temperature estimated at $1.4 \mathrm{GHz}$ from $F_{10.7}$ measurements conducted at Dominion Radio Astrophysical Observatory (DRAO), using the model developed by [13]. The available data at $1.4 \mathrm{GHz}$ show overall good agreement despite the different locations and instruments involved. An increasing dispersion is observed in the data with increasing sun activity, particularly, at the end of the selected period. This is to be expected since solar flux measurements from the few ground-based radiometers considered here were performed at specific hours and do not represent daily averaged fluxes. For the same day, radiometers may thus have captured different $R$-component flux values depending on the time at which measurements were performed. Nevertheless, Fig. 1 shows that the brightness temperature of the sun at $1.4 \mathrm{GHz}$ during 1996-2000, ranging from a minimum of about $1.1 \times 10^{5} \mathrm{~K}$ (the quiet sun brightness at $1.4 \mathrm{GHz}$ ) to about $9 \times 10^{5} \mathrm{~K}$. These numerical values will be used in the following as lower and upper limits for the sun brightness temperature expected at $1.4 \mathrm{GHz}$ during the SMOS mission period.

\section{Formulation of the Sun Glitter CONTRibutions at Surface LEVEL}

\section{A. Upwelling Sun Glitter Contributions at Surface Level}

Let us assume that an incremental terrestrial area $d A$ located within the radiometer antenna FOV is illuminated by the sun radiation along the direction of the unit vector $\vec{n}_{o}=\left(\theta_{o}, \Phi_{o}\right)$ at given time $t$. Part of the intercepted energy is scattered in the direction $\vec{n}_{s}=\left(\theta_{s}, \Phi_{s}\right)$, i.e., toward the radiometer antenna. The solar energy scattered by $d A$ in direction $\vec{n}_{s}$ at polarization $p$ and time $t$ is represented by radiometric temperature $T_{\mathrm{ss}}\left(\vec{n}_{s}, p, t\right)$. The sun brightness temperature incident in direction $\vec{n}_{o}$ at $t$ is denoted as $T_{\text {sun }}\left(\vec{n}_{o}, t\right)$ and the need to be corrected for attenuation along its path across the atmosphere. The upwelling horizontally and vertically polarized sun glint brightness temperature at surface level, which is hereafter 
denoted as bottom of atmosphere (BOA) contributions, can be related to $T_{\text {sun }}\left(\vec{n}_{o}, t\right)$ by the integral equations given in (4), where $\sigma_{\mathrm{hh}}^{\circ}, \sigma_{\mathrm{vv}}^{\circ}, \sigma_{\mathrm{vh}}^{\circ}$, and $\sigma_{\mathrm{hv}}^{\circ}$ are the bistatic scattering coefficients of the sea surface for $\mathrm{HH}, \mathrm{VV}, \mathrm{VH}$, and $\mathrm{HV}$ polarizations, respectively, at scattered direction $\vec{n}_{s}$ and incident direction $\vec{n}_{o}$. Thus we have

$$
\begin{aligned}
T_{\mathrm{ss}}^{\mathrm{BOA}}\left(\vec{n}_{s}, h, t\right)= & \frac{1}{4 \pi \cos \theta_{s}} \int_{\Omega_{\mathrm{sun}}}\left[\sigma_{\mathrm{hh}}^{\circ}\left(\vec{n}_{s}, \vec{n}_{o}\right)+\sigma_{\mathrm{hv}}^{\circ}\left(\vec{n}_{s}, \vec{n}_{o}\right)\right] \\
& \times T_{\mathrm{sun}}\left(\vec{n}_{o}, t\right) e^{-\tau \sec \theta_{o}} d \Omega_{o} \\
T_{\mathrm{ss}}^{\mathrm{BOA}}\left(\vec{n}_{s}, v, t\right)= & \frac{1}{4 \pi \cos \theta_{s}} \int_{\Omega_{\mathrm{sun}}}\left[\sigma_{\mathrm{vv}}^{\circ}\left(\vec{n}_{s}, \vec{n}_{o}\right)+\sigma_{\mathrm{vh}}^{\circ}\left(\vec{n}_{s}, \vec{n}_{o}\right)\right] \\
& \times T_{\mathrm{sun}}\left(\vec{n}_{o}, t\right) e^{-\tau \sec \theta_{o}} d \Omega_{o} .
\end{aligned}
$$

The notation is such that the first subscript refers to the polarization configuration of the scattered wave and the second subscript refers to that of the incident wave. $1-\tau$ is the atmospheric transmissivity at L-band; owing to its very weak impact on solar radiation at L-band, we neglect this damping atmospheric factor in our analysis.

Changes in solar radiation incidence direction $\vec{n}_{i}$ at given terrestrial targets over the solid angle subtended by the sun are small (within $\pm 0.25^{\circ}$ ) with respect to the synthesized angular resolution of SMOS' instrument $\left(2.25^{\circ}\right)$. Therefore, the sun appears as a point source, and considering the integrated brightness temperature signal radiated by the sun disc at time $t$, namely, $\bar{T}_{\text {sun }}(t)$, the sun glint brightness temperatures at surface level scattered in direction $\vec{n}_{s}$ and polarization $p$ can be further approximated by

$$
T_{\mathrm{ss}}^{\mathrm{BOA}}\left(\vec{n}_{s}, p, t\right) \simeq \frac{\bar{T}_{\mathrm{sun}}(t) \Omega_{\mathrm{sun}}}{4 \pi \cos \theta_{s}}\left[\sigma_{\mathrm{pp}}^{\circ}\left(\vec{n}_{s}, \vec{n}_{o}\right)+\sigma_{\mathrm{pq}}^{\circ}\left(\vec{n}_{s}, \vec{n}_{o}\right)\right] .
$$

\section{B. Bistatic Scattering Models at L-Band}

A key submodel in the sun glint evaluation is the model for the bistatic scattering coefficients of the rough sea surface at L-band that is needed in (5). As anticipated, the intensity and spread of the sun glint pollution pattern will depend not only on the measurement geometry but also on the sea surface roughness conditions. It was shown in [2] that the specularly reflected sun over a perfectly smooth Earth surface (i.e., no roughness) never intercepts the useful part of SMOS FOV. We further show that under general conditions, the expected pollution will come from contributions relatively far away from the pure specular directions, with local sun incidence angles always larger than $50^{\circ}$ when looking at targets inside the FOV. Knowing that SMOS multiangular capabilities range from $0^{\circ}$ to $60^{\circ}$, we seek to model scattering of radiation at directions varying almost from specular to grazing directions. Popular approximations in this context are the KA [9] and the SSA theory [7], [8]. The validity of KA approach is restricted to surfaces with large curvatures and to large Rayleigh parameters. The SSA has been proposed by Voronovich [7] as an alternative to efficiently bridge a small perturbation model (SPM) and KA models. In particular, SSA strictly meets SPM as the roughness goes to zero. Analytical expressions are in principle available for the SSA at all orders in slope. In practice, however, only the first two orders are tractable. The first-order SSA (SSA-1) implies the same single integral as in KA to determine a particular Fourier coefficient of the surface elevation coherence structure function with a different geometrical factor. The second-order approximation, however, is a double oscillating integral that is found to be very difficult to compute accurately, especially in the dielectric case where convergence problems and computational time demands become prohibitive. For this paper, we thus only consider SSA-1 and the Kirchhoff approaches to illustrate the potential impacts of the scattering models on the sun glint results.

To facilitate discussion, we introduce a local Cartesian coordinate system $(\hat{x}, \hat{y}, \hat{z})$, with $\hat{x}$ pointing eastward, $\hat{y}$ pointing northward, and basis vector $\hat{z}$ pointing upward normal to the horizontal surface.

Application of the SSA-1 or KA approximations for scattering from the slightly rough ocean surface yields the following expression for a dimensionless bistatic scattering cross section $\sigma_{\alpha \alpha_{o}}^{\circ}$ for the scattering of the incoming wave of polarization $\alpha_{o}$ into the outgoing wave of polarization $\alpha$ [14]:

$\sigma_{\alpha \alpha_{o}}\left(\vec{k}_{s}, \vec{k}_{o}\right)=\frac{1}{\pi}\left|\frac{2 q_{s} q_{o}}{q_{s}+q_{o}} B_{\alpha \alpha_{o}}\left(\vec{k}_{s}, \vec{k}_{o}\right)\right|^{2} e^{-\left(q_{s}+q_{o}\right)^{2} \rho(0)} \cdot I_{K}$

where $I_{K}$ is given in Cartesian coordinates by

$$
I_{K}=\iint\left\{e^{\left[\left(q_{s}+q_{o}\right)^{2} \rho(\vec{x})\right]}-1\right\} e^{\left[-i\left(\vec{k}_{s}-\vec{k}_{o}\right) \cdot \vec{x}\right]} d x d y
$$

where each integration ranges from $-\infty$ to $+\infty$ and where we use the notation $\vec{x}$ to denote the horizontal displacement vector and the integral is evaluated over all possible displacements in the horizontal plane. In (6) and $(7),\left(\vec{k}_{s}, \vec{k}_{o}\right)$ are the scattered and incident wavenumber vectors, $\left(q_{s}, q_{o}\right)=\left(\hat{z} \cdot \vec{k}_{s},-\hat{z} \cdot \vec{k}_{o}\right)$ represents the vertical projection of the wave vectors, and the kernel functions $B_{\alpha \alpha_{o}}\left(\vec{k}_{s}, \vec{k}_{o}\right)$ are geometric functions of the dielectric constant that differ in the SSA-1 approach compared to KA. Analytical expressions of these kernel functions can be found in [14] and [15]. We used the Klein and Swift's model [16] to estimate the dielectric constant of sea water at L-band.

If the sea surface elevation function is assumed to be a Gaussian random process, then $\rho$ represents the correlation function of the surface elevation and can be expressed strictly in terms of the inverse Fourier transform of the roughness spectrum, i.e.,

$$
\rho(x, y)=\int_{-\infty}^{\infty} \int_{-\infty}^{\infty} W\left(\xi_{x}, \xi_{y}\right) e^{i \vec{\xi} \cdot \vec{x}} d \xi_{x} d \xi_{y}
$$

where $W\left(\xi_{x}, \xi_{y}\right)$ is the directional wavenumber spectrum of the rough sea surface as a function of surface wavenumber vector in Cartesian number wavespace $\vec{\xi}=\left(\xi_{x}, \xi_{y}\right)$. Under the same hypothesis, $\rho$ is obtained from the inverse Fourier transform of the sea surface spectrum as computed using the spectral 

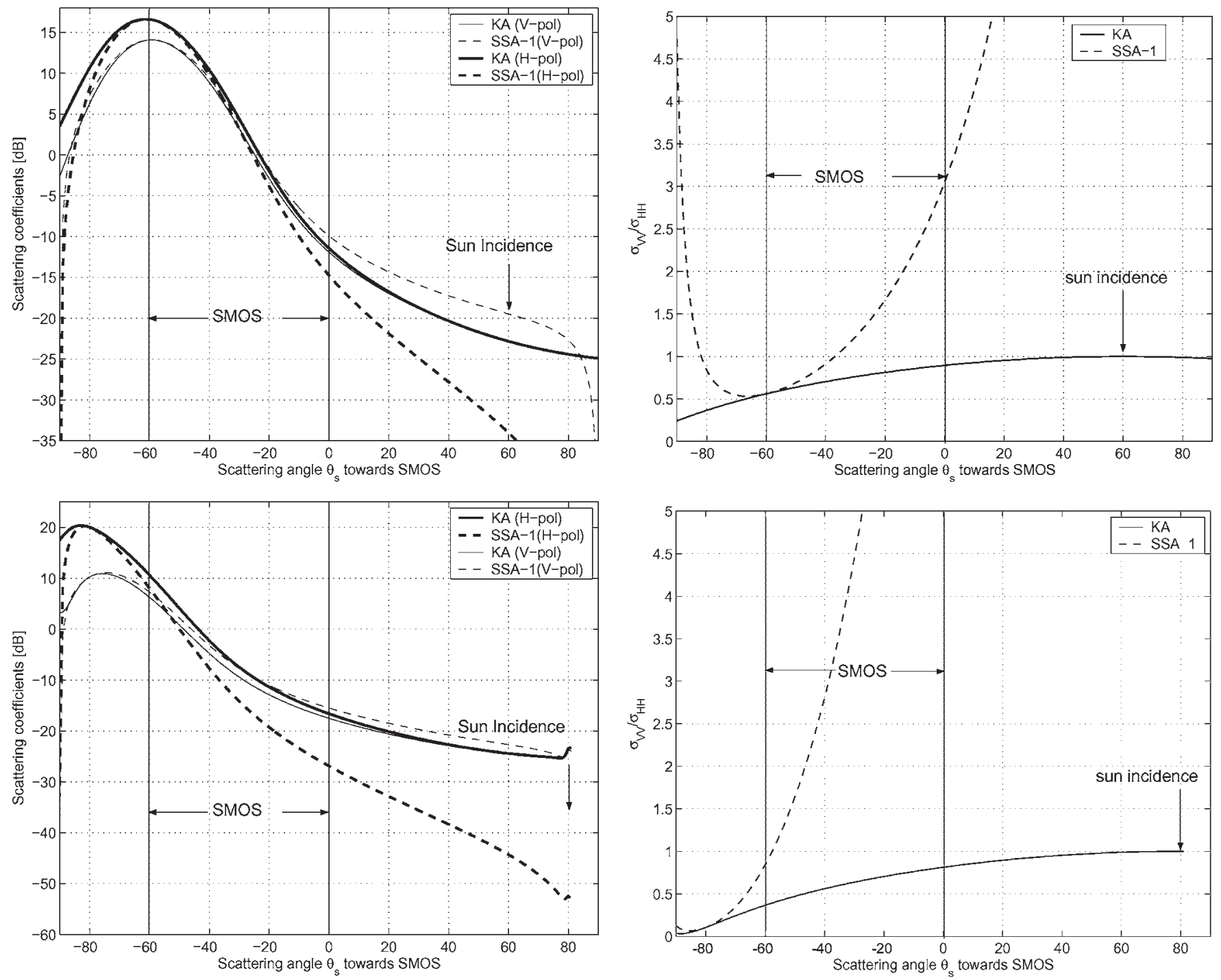

(a)

(b)

Fig. 2. Bistatic scattering coefficients and polarization ratio at L-band as a function of the scattering angles toward SMOS for two values of the sun incidence angle: $\theta_{o}=60^{\circ}$ (upper plots) and $\theta_{o}=80^{\circ}$ (lower plots), as evaluated with the SSA-1 and KA scattering models for a wind speed of $7 \mathrm{~m} / \mathrm{s}$, an SST of $15{ }^{\circ} \mathrm{C}$, and an SSS of 35 psu. The plots show sections of the 2-D bistatic scattering coefficients and the corresponding polarization ratio in the sun incidence plane. The convention is such that a minus sign in $\theta_{s}$ corresponds to a $180^{\circ}$ rotation with respect to the sun azimuth at the target. The range of potentially scattered directions toward SMOS is also indicated by two vertical lines. (a) Bistatic scattering coefficients. (b) Polarization ratio.

model of Kudryavtsev et al. [17]. Note that the second-order moment of that spectrum, namely, the integrated mean square slope parameter $(\mathrm{mss})$, was constrained to agree with Cox and Munk's [18], [19] clean and slick surface results. The choice for the sea surface spectrum model used in the calculation of the sun glint contribution is certainly an important issue for the sun glint model. However, the chosen spectrum follows physical principles and has been shown to provide consistent comparisons with both optical and active microwave measurements [20], [21].

In the Appendix, we show that the twofold integration (7) can be reduced to a 1-D integral using azimuthal harmonics decomposition of the integrand in polar coordinates and provide a fast computing method to estimate rough sea surface bistatic coefficients using either the small slope or the Kirchhoff asymptotic theory.
Examples of the bistatic scattering coefficients and associated polarization ratios calculated at L-band using both asympotic methods are shown in Fig. 2. The figures reveal the following.

- Differences between both models increase as the scattering direction moves away from the specular direction.

- Consequently, differences between model predictions for the range of scattering angles observed with SMOS will increase as the sun incidence angle increases.

- Near the specular direction, both models predict similar polarization ratio $\sigma_{\mathrm{vv}} / \sigma_{\mathrm{hh}}$.

- However, the polarization ratio predicted by KA is almost independent of the sun incidence angle in the range of scattering directions toward SMOS, and it never exceeds unity. 
- By contrast, the polarization ratio $\sigma_{\mathrm{vv}} / \sigma_{\mathrm{hh}}$, as predicted by SSA-1 for the SMOS range, is strongly dependent on both the sun incidence and scattering directions. It largely exceeds unity as scattering angles reach values greater than about $20^{\circ}$ away from specular.

As understood, these two approaches can be considered to provide the asymptotic limits within a consistent framework of scattering slope expansions. Both approaches somehow lack, taking into account directly the surface geometrical properties to predict the level of polarization. In particular, with the proposed models, the polarization levels are only given by the $B_{\alpha \alpha_{o}}$ kernels, which are themselves independent of roughness states. By construction, SSA-1 rapidly tends toward SPM at first order, which is known to certainly exaggerate the polarization dependencies [14], [15], while KA, as known to represent the high-frequency scattering limit, will minimize polarization effects.

\section{SUNGLint INTERACTIONS With SMOS}

In this section, the sun geometrical interactions with the SMOS antenna are first simulated for the first year of the mission, assuming the Earth surface to be locally perfectly smooth to illustrate expected variations in the respective position of the specular sun point and the SMOS FOV as a function of season and orbit position. Numerical simulations of the interaction between rough sea surface scattered sun radiation and SMOS are then performed for two characteristic days: the winter solstice (December 22) and the spring equinox (March 21), when the specular sun glint points assuming a perfectly smooth Earth surface were respectively found at the shortest and largest distances from the useful part of SMOS FOV.

\section{A. Seasonal Variations Assuming a Smooth Earth Surface}

SMOS will be placed in a circular sun-synchronous Low Earth Orbit at a mean flight altitude of $755 \mathrm{~km}$. The Local Time Ascending Node will be set at the equator at 06:00 A.M. The inclination of the orbit's plane with reference to the Earth's equatorial plane will be $98.42^{\circ}$. The SMOS satellite will be positioned at a pitch attitude such that the antenna plane will be tilted $32^{\circ}$ forward in flight direction.

We performed numerical simulations to track the seasonal variations of the sun location and of the specular sun reflection point, as observed in SMOS FOV. The sun angles (elevation $\theta_{\text {sun }}$ and azimuth $\Phi_{\text {sun }}$ ) are determined at each subsatellite point along the orbit, together with the azimuth of the satellite's orbit plane, namely, $\Phi_{\text {orb }}$. These angles are obtained with a sampling time rate of $14 \mathrm{~s}$ along the orbit, which corresponds to a sampling along the orbit ground track of about $100 \mathrm{~km}$. Fig. 3 illustrates the sun versus SMOS configuration for the example date January 18, 2007 at 23:16 UTC. A segment of the Earth track of SMOS' orbit during that day is shown. In this example, one have $\Phi_{\text {orb }}=194.3^{\circ}$, and $\theta_{\text {sun }}=21.2^{\circ}, \Phi_{\text {sun }}=261.4^{\circ}$ at the subsatellite point. It is seen that below SMOS, the sun is close to grazing (with an incidence angle of $\theta_{o}=90^{\circ}-\theta_{\text {sun }} \simeq$ $70^{\circ}$ ). Intersection between the vertical plane containing the sun

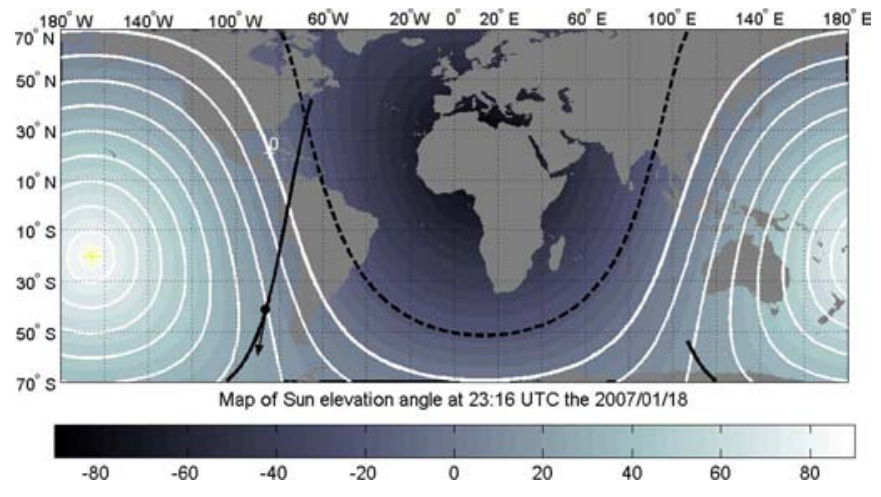

Fig. 3. Example of the global map of the solar elevation angles $\theta_{\text {sun }}$ at 23:16 UTC (January 18, 2007). The SMOS ground track is superimposed (black solid curve) from about $20 \mathrm{~min}$ before local time to about $20 \mathrm{~min}$ after local time. The black dot indicates subsatellite point at local time. The dark dashed curve indicates the astronomical twilight (the sun is $18^{\circ}$ below the horizon). The contour curve levels ranges from $-90^{\circ}$ to $90^{\circ}$ in steps of $10^{\circ}$.

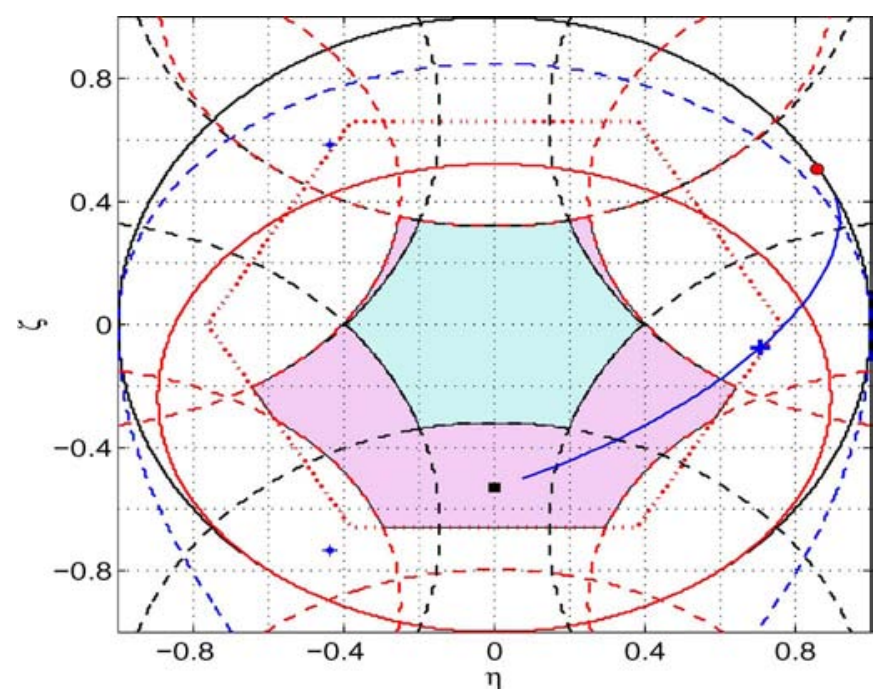

Fig. 4. Position of the sun for the example date January 18, 2007 at 23:16 UTC in the director cosine frame with coordinates $(\xi, \eta)$. The solid blue line is the track of the sun vertical plane in the antenna frame. The red large dot indicates the actual sun position. The large blue plus symbol indicates the specular sun glint point location. The small blue plus symbols show the aliases of that specular sun glint point. The cyan and pink areas are the alias and Earth AF FOV, respectively. The black square is the subsatellite point. The solid red and black curves are the Earth horizon and the $\left(\eta^{2}+\xi^{2}=1\right)$ boundary of the solid angle facing the antenna plane, respectively. The blue dotted curve is the extent of the horizontal plane in the geographical reference frame.

at the subsatellite point and the MIRAS antenna array plane is then determined by finding the director cosine coordinates of the antenna frame corresponding to the radiometer look azimuth equal to the sun azimuth angle plus $\pi$. Fig. 4 illustrates sun and sun glint position tracking in the antenna director cosine frame $(\xi, \eta)$ for the example date January 18, 2007 at 23:16 UTC.

The director cosine coordinates $(\xi, \eta)$ are defined as a function of angle $\left(\theta_{a}, \phi_{a}\right)$ in the antenna spherical coordinate system by

$$
\begin{aligned}
\xi & =\sin \theta_{a} \cos \phi_{a} \\
\eta & =\sin \theta_{a} \sin \phi_{a} .
\end{aligned}
$$


(a)

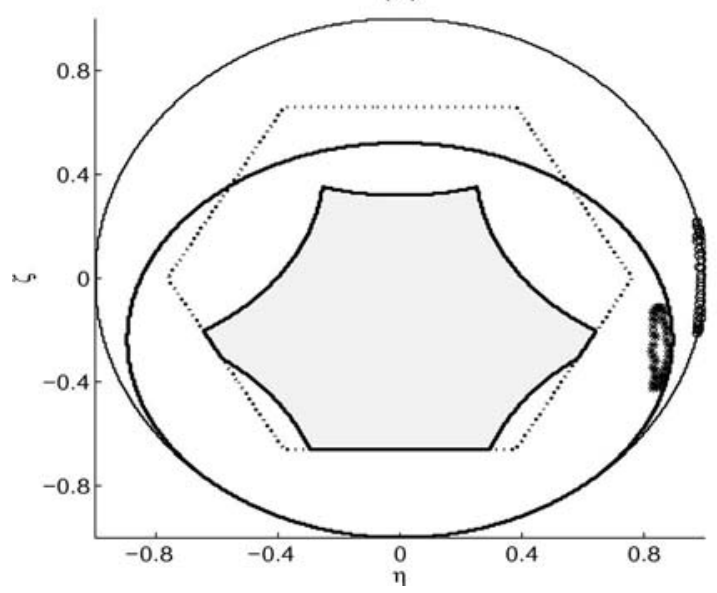

(b)

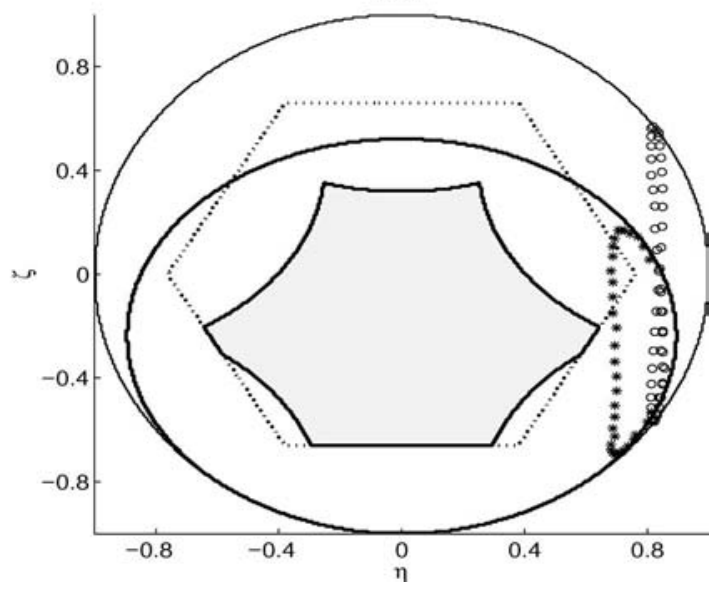

Fig. 5. Daily variations of the sun and specular sun glint locations as seen in the antenna frame of SMOS as a function of seasons. The large circles represent the sun location. The star symbols represent the location of the specular sun glint points. (a) Spring equinox. (b) Winter solstice.

The hexagonal sampling provided by a Y-shaped array that requires an antenna spacing of $d=1 / \sqrt{3}$ wavelengths to avoid aliasing. Due to technological limitations (antenna size and antenna mutual coupling), the minimum baseline in SMOS cannot be equal to $1 / \sqrt{3}$ but is rather $d \simeq 0.875$. The Nyquist criterion is therefore not satisfied, and there is aliasing. The alias-free (AF) FOV of the MIRAS interferometer is thus limited to the inner pixels of the region, which are defined by the periodic repetition of the unit circle centered at $(1 / \sqrt{3} d, 1 / d),(1 / \sqrt{3} d,-1 / d),(-1 / \sqrt{3} d, 1 / d)$, $(-1 / \sqrt{3} d,-1 / d),(2 / \sqrt{3} d, 0)$, and $(-2 / \sqrt{3} d, 0)$, which determines which pixels can be recovered without aliasing [22] (cyan-filled domain in Fig. 4). By accounting for sky contributions prior to the image reconstruction, it is possible to extend the AF FOV by including zones only contaminated by sky replicas; this defines the so-called "extended" or "Earth AF" FOV, which is represented by the pink-filled domain in Fig. 4. In the example given in Fig. 4, one can see that a specular sun glint event is occurring, but it is located outside the extended FOV, on the right-hand part of it. No alias of the sun can be observed in this case because the sun is located above the MIRAS antenna at this particular time.

Variations as a function of the seasonal cycle are illustrated in Fig. 5, where the daily track of the sun and that of the specular sun glint point locations are given for the date and time of winter solstice and spring equinox for year 2007. One single revolution of the satellite around Earth is considered per day in these plots. Indeed, very small changes are observed in the SMOS versus sun configuration between different revolutions for the same day. Fig. 5 illustrates that if the Earth surface were locally perfectly smooth, there would be no pollution by sun glint in the useful part of the FOV. However, as illustrated in Fig. 5(b), around the winter solstice, the specular sun glint point will sometimes be located close to it.

In Fig. 6, we plot the probability distribution functions of sun incidence angles at Earth target points within the Earth $\mathrm{AF}$ FOV for both days. It can be seen that the sun incidence at ocean targets within the Earth AF FOV of SMOS will be always larger than $50^{\circ}$.
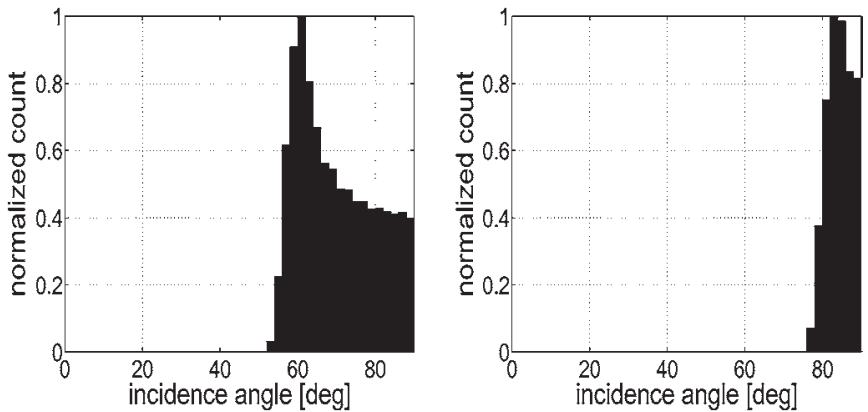

Fig. 6. Probability distribution functions of sun incidence angles at target points within the Earth AF FOV (left) for the winter solstice case and (right) for the spring equinox case (the histograms have been normalized to a maximum of unity).

\section{B. SMOS Measurements Pollution by Sun Glint in the Presence of Ocean Surface Roughness}

1) Numerical Simulation Description: Numerical simulations of the interaction between sun glint radiation and SMOS are performed for the two characteristic days, namely: 1) the winter solstice (December 22) and 2) the spring equinox (March 21), when the specular sun glint points were found at the shortest and largest distances from the useful part of SMOS FOV, respectively.

To assess the magnitude of the effect of roughness on sun glint, we performed a series of simulations, assuming uniform (constant) geophysical surface fields. The SSS and SST fields were fixed to their ocean average values of $35 \mathrm{psu}$ and $15{ }^{\circ} \mathrm{C}$, respectively. For the surface wind vector distributions, we successively conducted simulations using uniform winds at 3,7 , and $20 \mathrm{~m} / \mathrm{s}$ in order to illustrate the potential impact of low, moderate, and high wind speed situations, respectively. In the first series of simulations, the wind direction impact was not considered since we assumed isotropic sea surfaces (only the bistatic coefficient zeroth harmonic $\sigma_{\alpha \alpha_{o}}^{0}$ in (B2) is considered for the calculations).

To further evaluate the potential impact of the local roughness anisotropy on sun glint, actual values of wind directions 
were used in the second series of simulation. The wind forcing conditions over the ocean for the winter solstice and the spring equinox of year 2007 are thus simulated using wind direction fields from the daily QuikSCAT mean wind vector products for December 22, 2002 and March 21, 2003, which was chosen as the representative wind fields for both dates. These products are gap-filled and averaged synoptic wind fields reconstructed from discrete Level 2B SeaWinds products using an objective analysis method. They are provided on a $0.5^{\circ} \times 0.5^{\circ}$ grid by the IFREMER/Centre ERS d'Archivage et de Traitement (CERSAT/France).

Both previously described scattering theories (KA and SSA-1) were used to compute the forward scattering of sun microwave radiation in the two series of simulation.

The procedure used for simulating the sun glitter brightness temperature as seen from the MIRAS antenna for the two selected days is given as follows.

- Using SMOS orbit simulations, the subsatellite point geographical coordinates are calculated every 2 min along the orbit.

- Orbit segments that cover a complete sun versus SMOS interaction cycle are selected for each day. As illustrated in Fig. 7, these orbit's portions correspond approximately to 50 successive positions of the subsatellite point at 2-mn sampling.

- The cosine director coordinates $(\zeta, \eta)$ of the MIRAS antenna is mapped with a spatial step of $0.0062 \times 0.0062$ (which corresponds to a gridded map of $321 \times 321$ nodes in this frame). At each sampled time along the SMOS orbit, the latitude and longitude at all terrestrial targets within the MIRAS antenna cosinus director map are determined, taking into account the observation geometry.

- The SSA-1 and KA scattering models are applied at each terrestrial target to determine the local bistatic coefficients, using the local values of wind vector fields, as well as the observation geometry of the MIRAS antenna (local incidence and azimuth angles) and the sun incidence angles at the target considered.

- We chose a value of $T_{\text {sun }}=1.1 \times 10^{5} \mathrm{~K}$ (the quiet sun brightness temperature at $1.4 \mathrm{GHz}$ ) for our numerical simulations.

2) Impact of Isotropic Wind-Generated Rough Sea Surfaces: Representative contour plot examples of the sun glint contamination as predicted in the right-hand part of the MIRAS antenna, assuming a uniform surface wind speed of $7 \mathrm{~m} / \mathrm{s}$ during the spring equinox and winter solstice daily cycles, are given in Figs. 8 and 9, respectively. Each subplot corresponds to a particular subsatellite position selected along the orbit segments, as indicated in Fig. 7. Looking back at Fig. 2, it can be seen that the modeled bistatic scattering coefficients in the range of scattering angles toward SMOS are, in general, highest for the H-polarization using the KA model. Only the $\mathrm{H}$-polarization results for the KA model are therefore given in Figs. 8 and 9 to illustrate the maximum sun glint contamination expected for a given surface condition.

As illustrated, significant sun glint contamination can occur in the extended SMOS FOV due to scattering by the rough sea

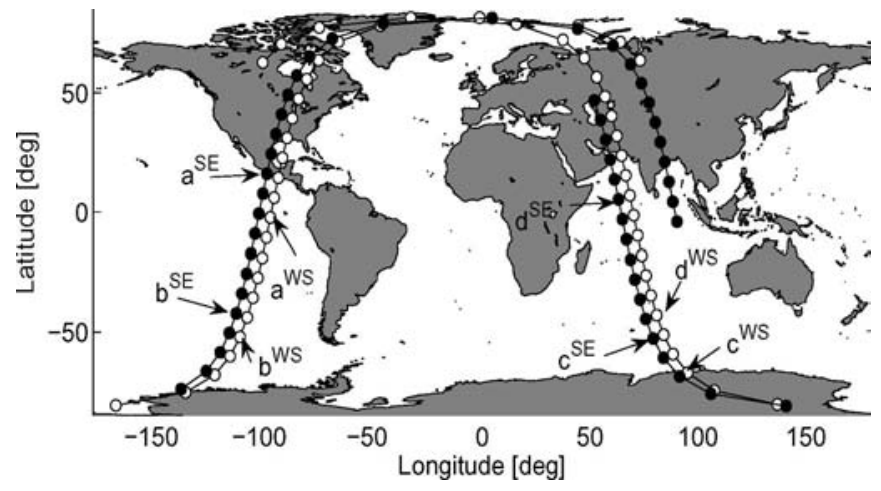

Fig. 7. Orbit segments of SMOS considered in our simulations for the spring equinox and winter solstice. The black filled dots indicate the sampled subsatellite point along the orbit (every $\simeq 2 \mathrm{~min}$ ) for the spring equinox. The black empty circles illustrate the sampling for the winter solstice. Positions corresponding to the example plots in Figs. 8 and 9 are shown by arrows and characters (a)-(d) with superscripts "SE" or "WS" to denote either "spring equinox" or "winter solstice" days, respectively.

surface. The closest the specular points from the border of the FOV, the more intense the contamination within the FOV due to interception of scattered contributions from quasi-specular directions. During the winter solstice, sun glint contamination can thus locally reach very significant levels with respect to the salinity signature at the L-band (up to about $50 \mathrm{~K}$ ) at a specific location along the orbit [e.g., Fig. 9(b)]. Conversely, when the specular points are located far away from the border of the extended FOV, sun glint contamination is weaker, with intercepted solar energy being scattered by the sea surface only from directions far away from the specular lobe. During the spring equinox (Fig. 8), the 1-K contour level is thus never observed to intersect the MIRAS extended FOV despite the same surface conditions than during the winter solstice. In some cases where the specular point is sufficiently far away from the FOV border, no significant contamination is observed [e.g., Fig. 8(d)].

To statistically assess the expected contamination variability along the orbit for each day, we calculated the mean, standard deviation, and maxima of the sun glint signals impinging the MIRAS extended FOV for each position along the orbit. The calculation was done for the three uniform wind conditions at 3,7 , and $20 \mathrm{~m} / \mathrm{s}$. Moreover, to evaluate the north/south distribution of contamination along the orbits, we further assumed here that the Earth surface observed by SMOS for the selected orbit segments is fully covered by ocean. The results for the mean and the maxima are illustrated in Fig. 10, showing explicitly that the contamination within the extended FOV will not be uniform along the orbit, being more intense when the satellite flies over the Southern Hemisphere where it exhibits clear maxima during the descending passes. As seen in Fig. 10(a) and (b), the average level of sun glint contamination is expected to be almost 20 times higher during the winter solstice than during the spring equinox with the maxima located at higher latitudes (around $65^{\circ}$ South) during the winter solstice than during the spring equinox (around $55^{\circ}$ South).

The stronger the surface wind, the higher the average contamination with a near doubling intensity from low wind conditions at $3 \mathrm{~m} / \mathrm{s}$ to high wind speeds at $20 \mathrm{~m} / \mathrm{s}$, where the maximum in average contamination can reach up to $0.5 \mathrm{~K}$ 


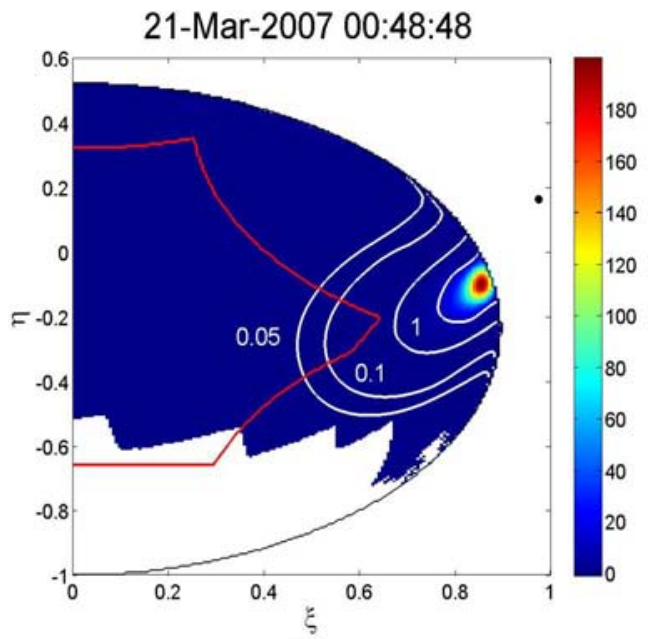

(a)

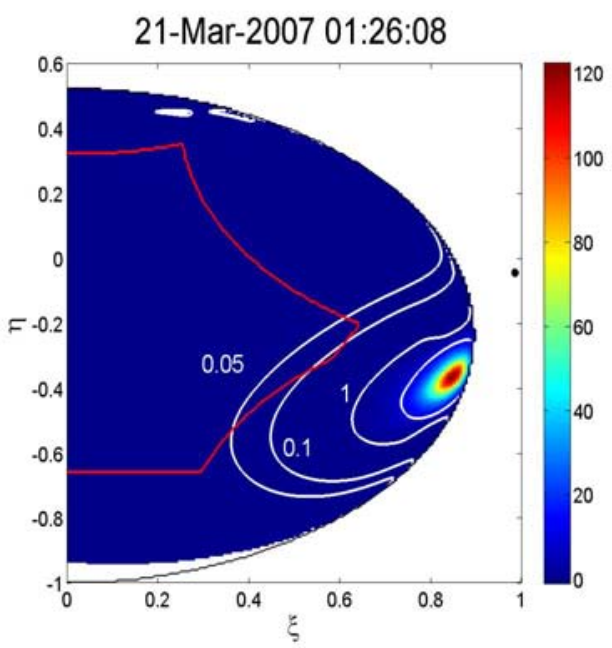

(c)

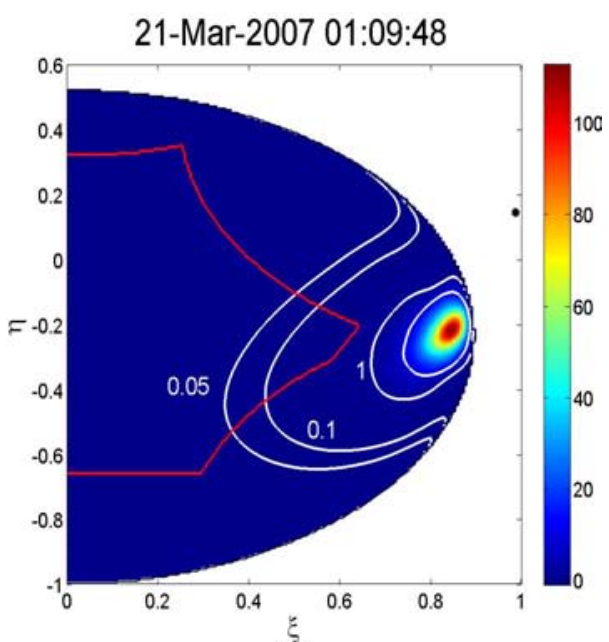

(b)

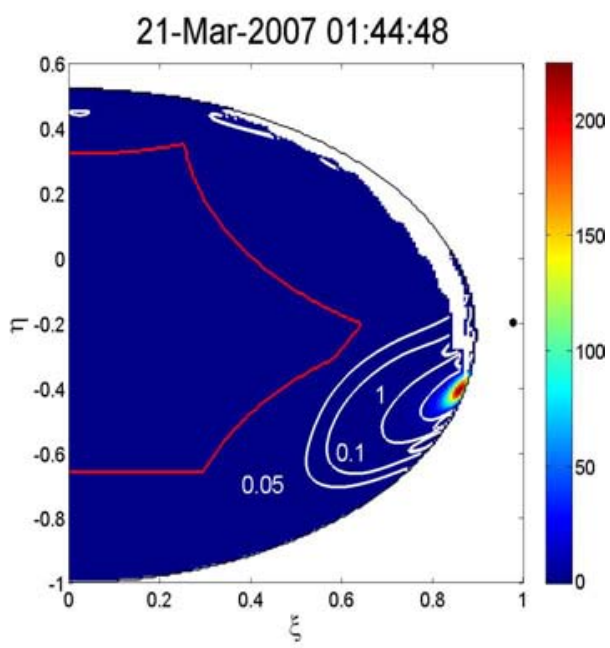

(d)

Fig. 8. Contour plots of the sun glint brightness temperature at the H-polarization (using the KA scattering model) superimposed with a color map of the sun glint intensity, as seen from the MIRAS antenna at different subsatellite positions during the spring equinox daily cycle, and assuming a uniform surface wind speed of $7 \mathrm{~m} / \mathrm{s}$ over the ocean. The contour levels are at $0.05,0.1,1,10$, and $100 \mathrm{~K}$. The black dots represents the sun position. The large white area illustrates the location of land surfaces. The threshold brightness temperature value of $0.05 \mathrm{~K}$, which represents about 0.1 psu at L-band (assuming an average sensitivity to SSS of about $0.5 \mathrm{~K} / \mathrm{psu}$ ), is used here as the lowest contour level to indicate significant contamination with respect to salinity impact.

during the winter solstice. Maximum sun glint signals within each FOV along the orbit for both days are given in Fig. 10(c) and (d). During the winter solstice, higher maxima are obtained for the low 3-m/s wind speed condition (around $50 \mathrm{~K}$ ), while during the spring equinox, higher maxima (around $1 \mathrm{~K}$ ) are obtained for the $20-\mathrm{m} / \mathrm{s}$ wind speed conditions. This is due to the specular sun glint point being closer from the FOV boundary during the winter solstice than during the spring equinox. Indeed, the lower (the higher) the wind speed, the higher the bistatic scattering coefficients in the vicinity of (far away from) the specular direction.

3) Impact of Anisotropic Wind-Generated Rough Sea Surfaces: To investigate the potential impact of surface roughness local directionality on the resulting sun glint brightness temperature predictions, we compared sun glint contamination estimated within the extended FOV, assuming surface fields defined by the following conditions:

- Condition 1(hereafter denoted C1): uniform surface wind speed fields generating isotropic roughness;
- Condition 2 (C2): uniform surface wind speed fields but with varying wind direction determined from QuikSCAT mean wind vector fields.

In Fig. 11(a), the contour levels of the sun glint contamination predictions for a uniform 7-m/s surface wind speed are plotted, assuming either isotropic (condition $\mathrm{C} 1$ ) or anisotropic roughness (condition $\mathrm{C} 2$ ), for one particular location along the orbit during the winter solstice. Anisotropy in the local surface roughness is observed to alter the shape of the sun glint pattern. In particular, if $0.05 \mathrm{~K}$ is chosen as a threshold level to detect data contaminated by sun glint, some of the SMOS data will be wrongly flagged and/or corrected, not accounting for the local wind direction impact.

The statistical impact of roughness anisotropy (or equivalently, of the wind direction variability) on sun glint estimates is illustrated in Fig. 11(b), where we show the mean differences in contamination within the FOV along the orbit between simulations assuming either isotropic (C1) or anisotropic (C2) rough surfaces, which are generated under the action of uniform 


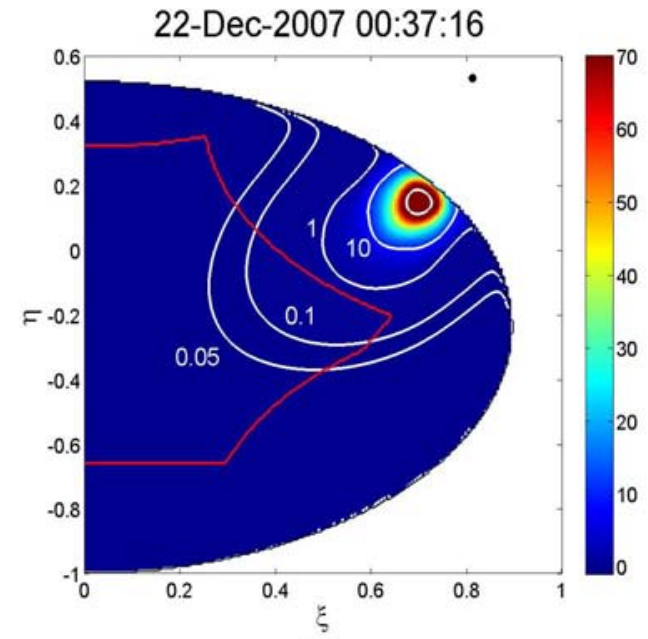

(a)

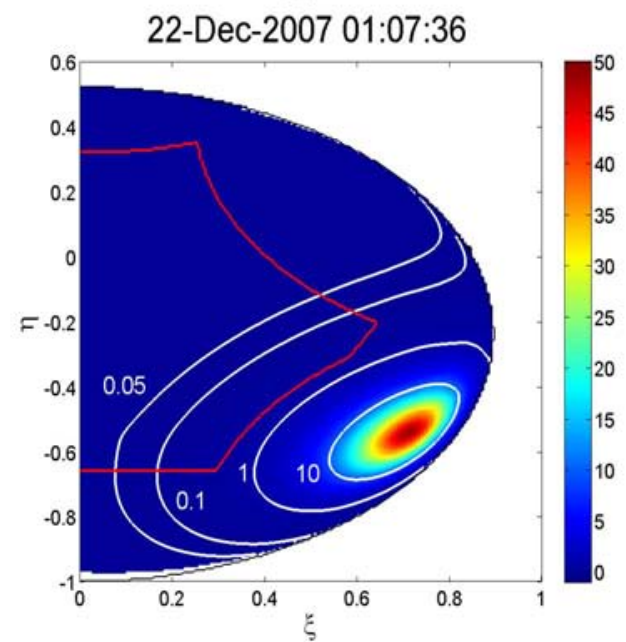

(c)

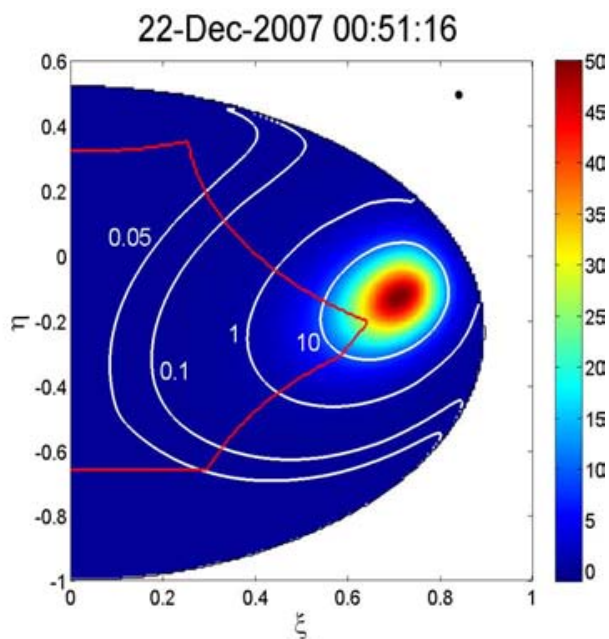

(b)

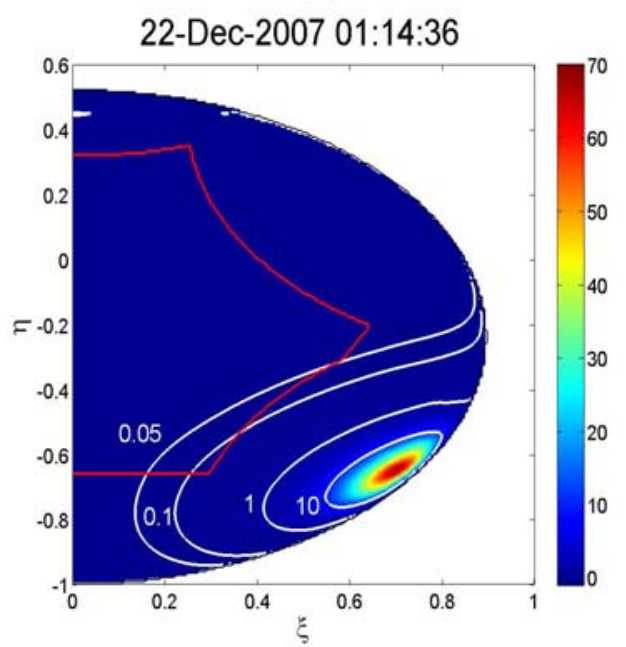

(d)

Fig. 9. Same as Fig. 8 but for the winter solstice.

wind speeds at either 3, 7, or $20 \mathrm{~m} / \mathrm{s}$. Same wind direction fields derived from QuikSCAT fields are used for the three different uniform wind speed conditions.

As shown, spatial variability in the wind direction alone may impact the predicted mean contamination, with an effect potentially greater than $\pm 0.05 \mathrm{~K}$ at specific locations along the orbit. The local wind direction impact will depend on the proximity of the sun specular point from the FOV border, on the surface wind vector distributions, and on the sun brightness temperature. In Fig. 11(b), the observed variability in the wind direction impact at a given location along the orbit as a function of wind speed can be explained as follows: Since the spatial extent of the significant contamination pattern is varying within the FOV as a function of wind speed, the wind direction distributions within the spatial domain that contributes to significant sun glint levels are also varying.

4) Impact of Electromagnetic Models: To evaluate the differences in sun glint predictions between the KA and SSA-1 approaches, statistical parameters characterizing the contamination were evaluated for each selected day using both models. These models were applied to actual heterogeneous surface wind vector fields provided by the QuikSCAT data to scan a larger range of simulated roughness states than to uniform wind fields. Daily average and maximum values of the brightness temperature pollution by sun glint at the $\mathrm{H}$ - and V-polarizations within SMOS FOV for the two selected days are given in Table I.

While scattering models exhibit similar pollution statistics at both polarizations during the winter solstice, they clearly differ during the spring equinox. This is a direct consequence of the differences in the statistics of the sun incidence angle at the targets (see Fig. 6), which exhibits higher values during the spring equinox than the winter solstice. Assuming a quiet sun brightness temperature, the observed average differences in the SMOS extended FOV for the winter solstice between estimated contributions from KA and SSA-1 models at the $\mathrm{H}$ - and V-polarizations are found to be 0.033 and $-0.050 \mathrm{~K}$, respectively, with similar standard deviations for both polarizations of order $0.17 \mathrm{~K}$. On average, the effect of choosing different scattering models is found to have negligible impact on the resulting sun glint pattern estimate (ten times smaller than the signal) for the cases where the specular reflected sun will be closest to the SMOS AF FOV. For the date of the spring equinox, the observed average differences between both 


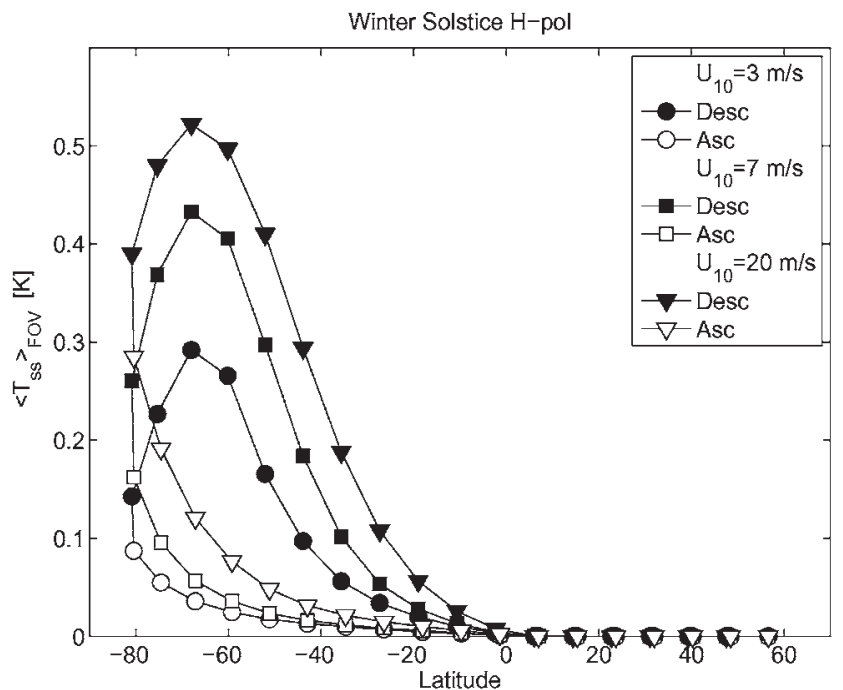

(a)

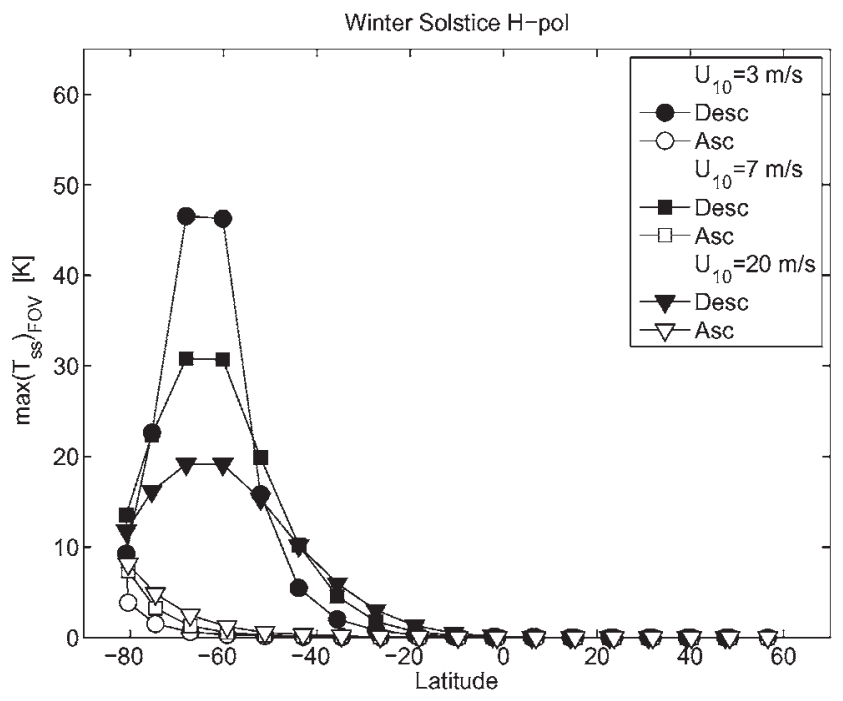

(c)

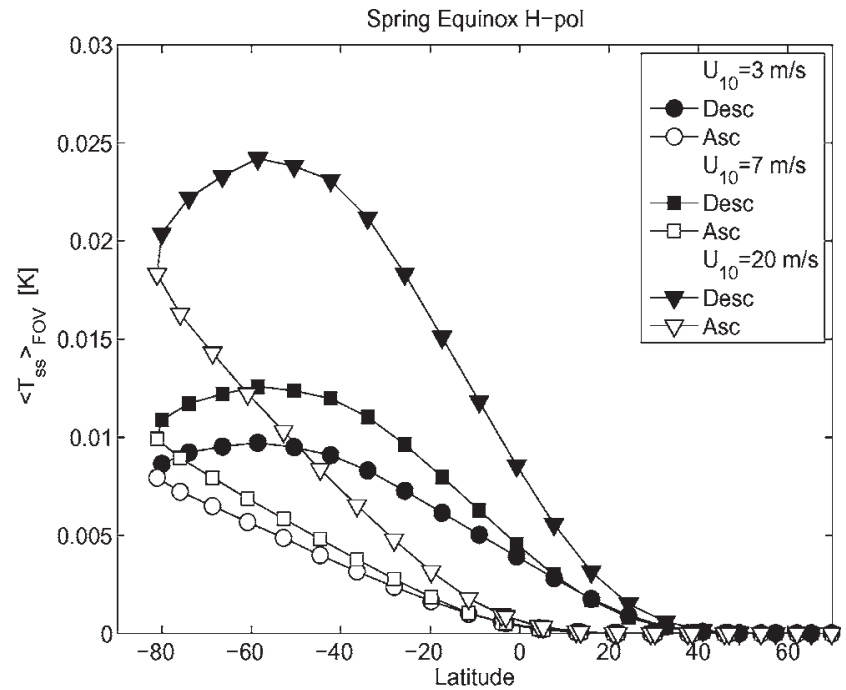

(b)

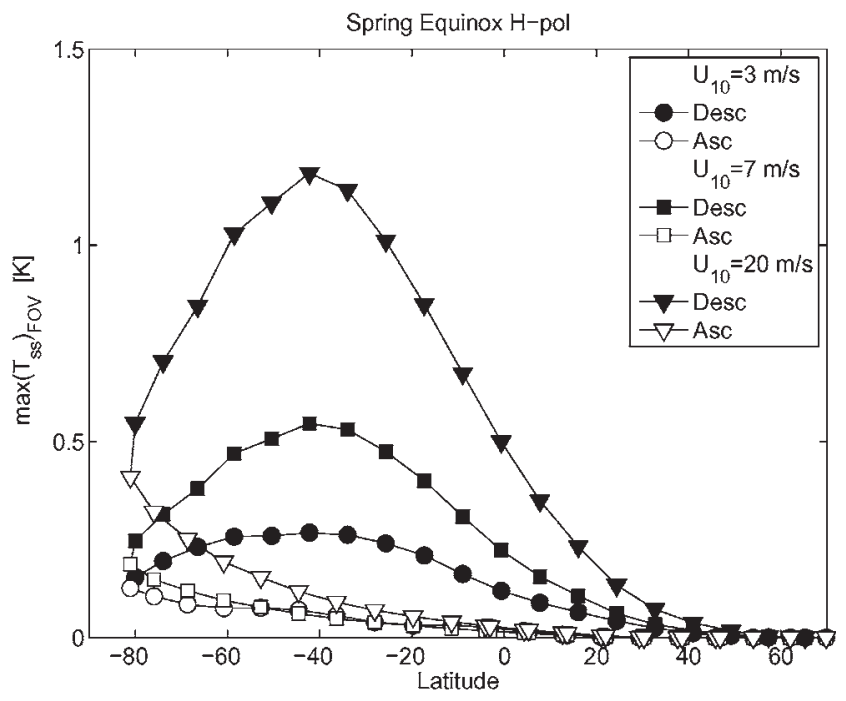

(d)

Fig. 10. Statistical characteristics of the sun glint contamination along the orbit as a function of the date (left plots: winter solstice; right plots: spring equinox) and as a function of the value of the uniform wind speed at a height of $10 \mathrm{~m}: 3 \mathrm{~m} / \mathrm{s}(\circ, \bullet), 7 \mathrm{~m} / \mathrm{s}(\square, \boldsymbol{\square})$, and $20 \mathrm{~m} / \mathrm{s}(\nabla, \boldsymbol{\nabla})$. Empty symbols are used to denote ascending passes, while filled symbols are used for descending passes. The upper and lower plots are showing the mean and maxima of the sun glint contamination within the FOV, respectively, at the H-polarization and using the KA scattering model. (a) Average contamination. (b) Average contamination. (c) Maxima of contamination. (d) Maxima of contamination.

models at the $\mathrm{H}$ - and V-polarizations are found to be 0.016 and $-0.007 \mathrm{~K}$, respectively. The corresponding standard deviations of differences are found to be 0.036 and $0.092 \mathrm{~K}$. The effect of choosing different scattering models is found to be more important, but the situation is representative of a minimum sun glint impact.

Note that all statistical results presented in this paper were obtained using the quiet sun brightness temperature value at $1.4 \mathrm{GHz}\left(T_{\text {sun }} \simeq 10^{5} \mathrm{~K}\right)$. During the winter solstice, the maximum expected pollution can reach about $50 \mathrm{~K}$, while they do not exceed $1.5 \mathrm{~K}$ during the spring equinox. For a strong solar activity, $T_{\text {sun }}$ can reach $\simeq 9 \times 10^{5} \mathrm{~K}$ so that according to (5), the sun glint leakage intensity, as given in Table I, should be multiplied by about a factor of ten to reach potential maximum values of up to $500 \mathrm{~K}$.

\section{DISCUSSION AND CONCLUSION}

In this paper, our understanding of the solar radiation features at $1.4 \mathrm{GHz}$ was reviewed, and an asymptotic forward scattering model of the sun glint contributions at L-band was presented. In order to test the sensitivity of the sun glint model results to the rough sea surface bistatic scattering coefficient models at L-band, two differing asymptotic scattering theories were used, namely: 1) the SSA-1 theory [7], [8] and 2) the Kirchhoff [9] approximation, both of which are applied to the same sea surface roughness spectrum model. Beyond these generic steps, this paper next focused on the specific case of the future SMOS mission.

Past ground-based measurements of the solar radio fluxes at $1.4 \mathrm{GHz}$ were used together with their reported 11-year periodicity to derive a potential time series of the sun brightness 


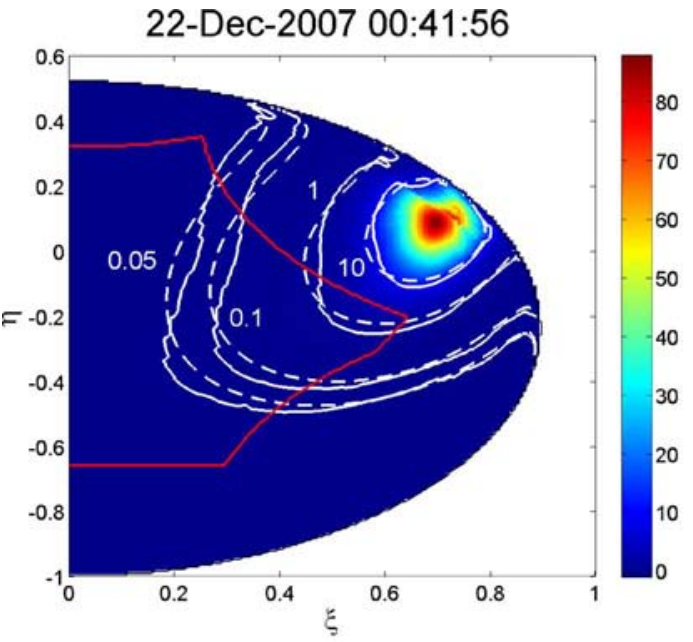

(a)

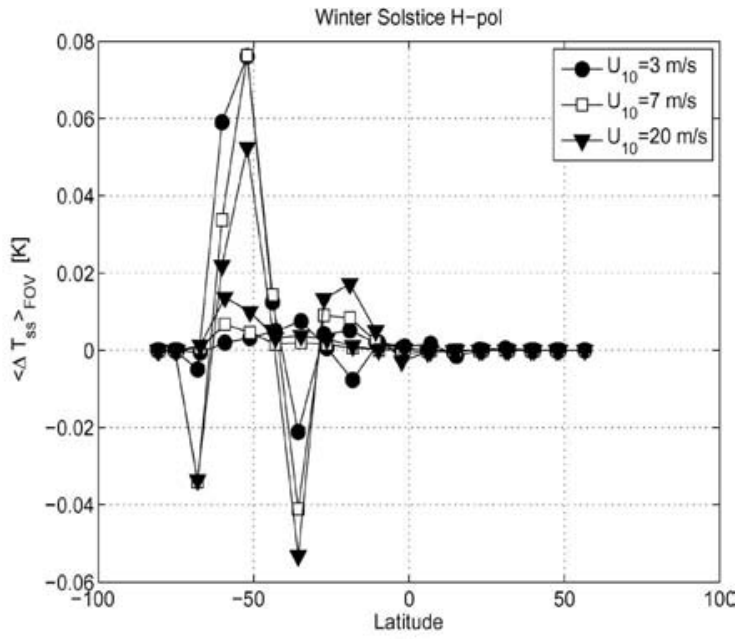

(b)

Fig. 11. Impact of surface roughness anisotropy on the sun glint contamination. (a) Contour levels of the sun glint contamination predictions for a uniform 7-m/s surface wind speed assuming either isotropic (dashed white curves) or anisotropic roughness (solid white curves) for one particular location along the orbit during the winter solstice. The contour levels are at $0.05,0.1,1$, and $10 \mathrm{~K}$. (b) Mean differences between sun glint contamination predictions along the orbit assuming isotropic and anisotropic roughness for uniform low, moderate, and high wind speed conditions. For the anisotropic roughness, the wind direction is obtained from actual QuikSCAT fields.

TABLE I

Daily AVerage, Standard, and Maximum Values of BRIGHTNESS TEMPERATURE POLLUTION BY SUNGLINT

\begin{tabular}{|c||c|c||c|c|}
\hline Winter Solstice & KA-H & KA-V & SSA1-H & SSA1-V \\
\hline \hline Mean $\bar{T}_{s s}[K]$ & 0.35 & 0.25 & 0.32 & 0.29 \\
\hline$\sigma_{T_{s s}}[K]$ & 1.79 & 1.2 & 1.6 & 1.28 \\
\hline $\max \left(T_{s s}\right)[\mathrm{K}]$ & 51 & 34.1 & 50.5 & 34.3 \\
\hline Spring Equinox & KA-H & KA-V & SSA1-H & SSA1-V \\
\hline \hline Mean $\bar{T}_{s S}[K]$ & 0.033 & 0.023 & 0.017 & 0.030 \\
\hline$\sigma_{T_{s s}}[K]$ & 0.062 & 0.036 & 0.039 & 0.098 \\
\hline $\max \left(T_{s s}\right)[\mathrm{K}]$ & 1.26 & 0.720 & 0.950 & 1.37 \\
\hline
\end{tabular}

temperature at $1.4 \mathrm{GHz}$ for the future SMOS mission period. The sun brightness temperature expected at $1.4 \mathrm{GHz}$ during the mission period will range from a quiet sun level of $1.1 \times 10^{5} \mathrm{~K}$ to maximum values of about $9 \times 10^{5} \mathrm{~K}$. The next solar maximum is expected in year 2010, and the sun glint contamination amplitude will continuously increase during the first three years of the mission lifetime.

Differences between asymptotic scattering theories were analyzed in the SMOS context. The sun is incident at Earth targets within SMOS' FOV, with angles ranging from about $60^{\circ}$ to grazing, and the radiometer is receiving scattered radiation at angles that are, in general, larger than $10^{\circ}$ away from the specular direction. For this range of bistatic angles, the SSA-1 model shall exaggerate the polarization dependencies, while KA will definitively minimize them.

The impact of the indirect leakage by sun glint on a simulated rough sea surface was estimated for two selected characteristic days of the sun seasonal cycle (winter solstice and spring equinox) corresponding to the maximum and minimum expected impacts. To assess the magnitude of the effect of sun glint, we first assumed uniform (constant) surface wind fields and made calculations for the global ocean average wind speed condition $(7 \mathrm{~m} / \mathrm{s}$ ) and for two extremes (low wind and high wind conditions) with everything else constant. Only isotropic rough sea surfaces were considered in these first series of simu- lations. We show that sun glint contamination within the righthand part of the MIRAS Earth AF FOV in the presence of variable wind speed might occur with an intensity ranging from $0 \mathrm{~K}$ to about $500 \mathrm{~K}$, depending on the target position in the polluted area, the season period, the roughness state at the target, and the level of solar activity at the time of measurements. The impact of local roughness anisotropy was then estimated by calculating the sun glint contamination for uniform wind speeds but with varying local directions derived from representative QuikSCAT products. Considering the sun brightness temperature level to be the quiet sun, the impact of anisotropy will be small with respect to the salinity signature, although it can locally exceed $\pm 0.05 \mathrm{~K}$. Three years after the launch, the slow varying components of the sun will reach an average brightness temperature level in the order of $3-4 \times 10^{5}$ (see Fig. 1). For such solar activity levels, the roughness anisotropy impact will potentially reach about $\pm 0.2 \mathrm{~K}$, which is significant with respect to the salinity signature, and data will be wrongly flagged and/or corrected, not accounting for the local wind direction impact. This suggests that the wind direction impact will have to be considered in the sun glint data processing for SMOS.

Potential errors in the sun glint modeling are certainly expected. More importantly, the knowledge of both the sea surface roughness states in SMOS pixels and the brightness temperature of the sun is crucial to determine the exact spread of the sun pollution. As most of the incoming solar radiation is close to grazing, shadowing effects not accounted for in our model might occur and create additional uncertainty. However, according to the given choice of surface wave spectrum in our model, the results show relatively small differences between KA and SSA-1 predictions, assuming a quiet sun brightness temperature value. This shows that the important contributions can be mostly attributed to the spread of the so-called quasispecular lobe. The use of the Kirchhoff method is known to be robust at L-band and to include filtered Geometric Optics solutions [23]. This can help validation exercises, comparing the 
spread of the modeled sun glint pollution to real future polluted data. The surface wave spectral model can be, in particular, further constrained in terms of effective mean squared slopes with known global positioning system L-band bistatic data (e.g., [24]).

As shown in Yueh et al. [1], the brightness temperature sensitivity to SSS at L-band ranges from 0.2 to $0.6 \mathrm{~K} / \mathrm{psu}$ for the $\mathrm{H}$-polarization and from 0.35 to $0.8 \mathrm{~K} / \mathrm{psu}$ for the V-polarization. Therefore, our results confirm that the sun glint impact is clearly a significant source of error for SSS remote sensing from SMOS, even when quiet sun temperature is considered over moderately wind-roughened surfaces. In SMOS Level-1 and Level-2 data processing, sun glint corrections and flags will be defined based on the forward model presented in this paper.

\section{APPENDIX \\ EFFICIENT IMPLEMENTATION OF BISTATIC SCATTERING COEFFICIENTS}

We review the method we used for bistatic scattering coefficients efficient numerical implementation. First, the Kirchhoff integral (7) is analytically expressed in polar coordinates so that the bistatic scattering coefficients dependence on wind direction is separated from the dependencies on other variables, without introducing further approximations. This allows calculation of bistatic scattering coefficients using a single numerical integration over the radial distance instead of two, as with a Cartesian coordinate formulation, saving a large amount of processing time.

Second, we make use of precalculated lookup tables (LUTs) of the bistatic scattering harmonic coefficients to provide a fast computing method to estimate rough sea surface bistatic coefficients using either the small slope or the Kirchhoff asymptotic theory.

\section{A. Kirchhoff Integral in Polar Coordinates}

The Kirchhoff integral can be written in polar coordinates as follows:

$$
I_{K}=\int_{0}^{\infty} \int_{0}^{2 \pi}\left\{e^{\left[\left(q_{s}+q_{o}\right)^{2} \rho(\vec{r})\right]}-1\right\} e^{-i\left(\vec{k}_{s}-\vec{k}_{o}\right) \cdot \vec{r}} d \vec{r}
$$

where $\left(\vec{k}_{s}, \vec{k}_{o}\right)$ are the scattered and incident wavenumber vectors; $\left(q_{s}, q_{o}\right)=\left(\hat{z} \cdot \vec{k}_{s},-\hat{z} \cdot \vec{k}_{o}\right)$ represents the vertical projection of the wavevectors, with $\hat{z}$ being a unit vector normal to the surface at the target and directed upward; $(r, \Phi)=\vec{r}$ are the polar coordinates; and $\rho(\vec{r})$ is the sea surface elevation autocorrelation function. The Fourier series expansion of $\rho(\vec{r})$ in azimuthal harmonics up to the second order can be expressed as follows [25], [26]:

$$
\rho(r, \Phi)=\rho_{0}(r)-\rho_{2}(r) \cos 2\left(\Phi-\varphi_{w}\right)
$$

where $\varphi_{w}$ is the wind direction and the isotropic $\rho_{0}(r)$ and anisotropic $\rho_{2}(r)$ parts are given by

$$
\left\{\begin{array}{l}
\rho_{0}(r)=\int_{0}^{\infty} W(k) J_{0}(r k) d k \\
\rho_{2}(r)=\int_{0}^{\infty} W(k) \Delta(k) J_{2}(r k) d k
\end{array}\right.
$$

where $k$ is a surface wavenumber, $J_{n}$ is the $n$ th-order Bessel function of the first kind, $W(k)$ is the omnidirectional sea surface height spectrum at wavenumber $k$, and $\Delta(k)$ is the socalled upwind-crosswind ratio at wavenumber $k$ [26].

In the following, we denote as $\vec{q}=\vec{k}_{s}-\vec{k}_{o}=q_{\mathrm{Hx}} \hat{x}+$ $q_{\mathrm{Hy}} \hat{y}+q_{z} \hat{z}$ the vector defined as the difference between the scattered and incident electromagnetic wavenumbers. We further denote as $q_{H}=\left|q_{\mathrm{Hx}} \hat{x}+q_{\mathrm{Hy}} \hat{y}\right|$ the horizontal component of $\vec{q}$ and introduce the angle $\Phi_{\mathrm{si}}=\tan ^{-1}\left(q_{\mathrm{Hy}} / q_{\mathrm{Hx}}\right)$.

Substituting (A2) into (A1), the Kirchhoff integral in polar coordinates becomes

$$
\begin{array}{r}
I_{K}=\int_{0}^{\infty} \int_{0}^{2 \pi}\left\{e^{\left[q_{z}^{2} \rho_{0}(r)-q_{z}^{2} \rho_{2}(r) \cos 2\left(\Phi-\varphi_{w}\right)\right]}-1\right\} \\
e^{-i q_{H} r \cos \left(\Phi-\Phi_{\mathrm{si}}\right)} r d \Phi d r .
\end{array}
$$

Gathering the terms functions of $\Phi$ in (A4), we have

$$
I_{K}=2 \pi \int_{0}^{\infty}\left[I_{\Phi_{1}}(r) e^{q_{z}^{2} \rho_{0}(r)}-I_{\Phi_{2}}(r)\right] r d r
$$

where we need to solve the following integrals over $\Phi$ :

$$
\left\{\begin{array}{l}
I_{\Phi_{1}}(r)=\frac{1}{2 \pi} \int_{0}^{2 \pi} e^{-a(r) \cos 2\left(\Phi-\varphi_{w}\right)-i b(r) \cos \left(\Phi-\Phi_{\mathrm{si}}\right)} d \Phi \\
I_{\Phi_{2}}(r)=\frac{1}{2 \pi} \int_{0}^{2 \pi} e^{-i b(r) \cos \left(\Phi-\Phi_{\mathrm{si}}\right)} d \Phi
\end{array}\right.
$$

where $a(r)=q_{z}^{2} \rho_{2}(r)$ and $b(r)=q_{H} r$. The complex exponential in (A6) can be expressed in terms of Bessel functions using the Jacobi-Anger expansion (see, e.g., [27]), i.e.,

$$
\begin{aligned}
e^{-a \cos 2\left(\Phi-\varphi_{w}\right)} & =\sum_{m=-\infty}^{\infty} i^{m} J_{m}(i a) e^{2 i m\left(\Phi-\varphi_{w}\right)} \\
& =J_{0}(i a)+2 \sum_{m=1}^{\infty} i^{m} J_{m}(i a) \cos 2 m\left(\Phi-\varphi_{w}\right)
\end{aligned}
$$

and

$$
\begin{aligned}
e^{-i b \cos \left(\Phi-\Phi_{\mathrm{si}}\right)} & =\sum_{n=-\infty}^{\infty} i^{n} J_{n}(b) e^{i n\left(\Phi-\Phi_{\mathrm{si}}+\pi\right)} \\
& =J_{0}(b)+2 \sum_{n=1}^{\infty} i^{3 n} J_{n}(b) \cos n\left(\Phi-\Phi_{\mathrm{si}}\right)
\end{aligned}
$$

where $J_{m}$ is the Bessel function of the first kind and order $m$. Substituting (A7) and (A8) into (A6) and performing the 
integration over $\Phi$, we obtain

$$
\begin{aligned}
I_{\Phi_{1}}(r)=J_{0}(i a) & J_{0}(b)+2 \sum_{m=1}^{\infty} \sum_{n=1}^{\infty} \delta(2 m-n) \\
& \times J_{m}(i a) J_{n}(b) i^{m+3 n} \cos n\left(\Phi_{\mathrm{si}}-\varphi_{w}\right)
\end{aligned}
$$

where $\delta$ is the Kronecker Delta. Using the relation $J_{m}(i a)=$ $i^{m} I_{m}(a)$, where $I_{m}$ denotes the modified Bessel function of the first kind and order $m$, we obtain

$I_{\Phi_{1}}(r)=I_{0}(a) J_{0}(b)+2 \sum_{m=1}^{\infty} I_{m}(a) J_{2 m}(b) \cos 2 m\left(\Phi_{\mathrm{si}}-\varphi_{w}\right)$.

For $I_{\Phi_{2}}(r)$, we have

$$
\begin{aligned}
I_{\Phi_{2}}(r) & =\frac{1}{2 \pi} \int_{0}^{2 \pi}\left[J_{0}(b)+2 \sum_{n=1}^{\infty} i^{n} J_{n}(b) \cos n\left(\Phi-\Phi_{\mathrm{si}}\right)\right] d \Phi \\
& =J_{0}(b) .
\end{aligned}
$$

Substituting (A10) and (A11) into (A5), the Kirchhoff integral reads

$$
I_{K}=\sum_{m=0}^{\infty} I_{K}^{m} \cos 2 m\left(\Phi_{\mathrm{si}}-\varphi_{w}\right)
$$

where

$$
I_{K}^{0}=2 \pi \int_{0}^{\infty} J_{0}\left(q_{H} r\right)\left[I_{0}\left(q_{z}^{2} \rho_{2}(r)\right) e^{q_{z}^{2} \rho_{0}(r)}-1\right] r d r
$$

and for $m$ from 1 to $\infty$

$$
I_{K}^{m}=4 \pi \int_{0}^{\infty} I_{m}\left(q_{z}^{2} \rho_{2}(r)\right) J_{2 m}\left(q_{H} r\right) e^{q_{z}^{2} \rho_{0}(r)} r d r
$$

is the coefficient of harmonic $2 m$ in a cosine series decomposition of the Kirchhoff integral.

Equation (A12) for the Kirchhoff integral allows calculation of bistatic scattering coefficients using a single numerical integration over the radial distance instead of four, as with a Cartesian coordinate formulation.

\section{B. Numerical Implementation}

Recalling the expression for the bistatic scattering coefficients

$$
\sigma_{\alpha \alpha_{o}}\left(\vec{k}_{s}, \vec{k}_{o}\right)=\frac{1}{\pi}\left|\frac{2 q_{s} q_{o}}{q_{s}+q_{o}} B_{\alpha \alpha_{o}}\left(\vec{k}_{s}, \vec{k}_{o}\right)\right|^{2} e^{-\left(q_{s}+q_{o}\right)^{2} \rho(0)} \cdot I_{K}
$$

we see that we can incorporate the polarization-dependent coefficients multiplying the Kirchhoff integral, Ik, in (B1) into the sum (A12) over the Kirchhoff integral harmonics. We thus obtain harmonic decompositions of the bistatic scattering coefficients, i.e.,

$$
\sigma_{\alpha \alpha_{o}}\left(\vec{k}_{s}, \vec{k}_{o}, u_{10}, \varphi_{w}\right)=\sum_{m=0}^{\infty} \sigma_{\alpha \alpha_{o}}^{m}\left(\vec{k}_{s}, \vec{k}_{o}\right) \cos 2 m\left(\Phi_{\mathrm{si}}-\varphi_{w}\right)
$$

where we have explicitly included the dependence of the final scattering coefficients on wind speed $u_{10}$ and wind direction $\varphi_{w}$, and where

$\sigma_{\alpha \alpha_{o}}^{m}\left(\vec{k}_{s}, \vec{k}_{o}\right)=\frac{1}{\pi}\left|\frac{2 q_{s} q_{o}}{q_{s}+q_{o}} B_{\alpha \alpha_{o}}\left(\vec{k}_{s}, \vec{k}_{o}\right)\right|^{2} e^{-\left(q_{s}+q_{o}\right)^{2} \rho(0)} \cdot I_{K}^{m}$.

Note that the scattering coefficient harmonics are independent of wind direction. Moreover, these harmonics only depend on the incoming and scattered radiation incidence angles, the wind speed, the dielectric constant, and the difference between the incoming and scattered radiation azimuth angles. Thus, switching from vector notation to angles, we can write the scattering coefficients as

$$
\begin{aligned}
& \sigma_{\alpha \alpha_{o}}\left(\theta_{o}, \phi_{o}, \phi_{s}, \theta_{s}, u_{10}, \varphi_{w}\right) \\
& \quad=\sum_{m=0}^{\infty} \sigma_{\alpha \alpha_{o}}^{m}\left(\theta_{o}, \phi_{s}-\phi_{o}, \theta_{s}, u_{10}\right) \cos 2 m\left(\Phi_{\mathrm{si}}-\varphi_{w}\right)
\end{aligned}
$$

where $\theta_{o}$ is the incoming radiation incidence angle, $\phi_{s}$ is the scattered radiation azimuth angle, $\phi_{o}$ is the incoming radiation azimuth angle, $\theta_{s}$ is the scattered radiation incidence angle. As defined previously, $\varphi_{w}$ is the wind direction (toward which the wind is blowing), and $\Phi_{\mathrm{si}}\left(\theta_{o}, \phi_{o}, \phi_{s}, \theta_{s}\right)=$ $\tan ^{-1}\left(\left(\sin \theta_{s} \sin \phi_{s}+\sin \theta_{o} \sin \phi_{o}\right) /\left(\sin \theta_{s} \cos \phi_{s}+\right.\right.$ $\left.\left.\sin \theta_{o} \cos \phi_{o}\right)\right)$ is a function of the incidence and azimuth angles of the incoming and scattered radiation.

We precalculate the LUTs of the harmonic coefficients $\sigma_{\alpha \alpha_{o}}^{m}\left(\theta_{o}, \phi_{s}-\phi_{o}, \theta_{s}, u_{10}\right)$ for even azimuthal wavenumbers $0-10$ on a discrete grid and interpolate from that grid. As the final step, the sum is implemented over the harmonics, given specific values for the incoming and scattered azimuths and wind direction. As it has been found that only the first few harmonics contribute significantly to the scattering coefficients, the sum is over the first six even harmonics.

\section{ACKNOWLEDGMENT}

The authors would like to thank the reviewers for helping to improve the manuscript.

\section{REFERENCES}

[1] S. H. Yueh, R. West, W. J. Wilson, F. K. Li, E. G. Njoku, and Y. Rahmat-Samii, "Error sources and feasibility for microwave remote sensing of ocean surface salinity," IEEE Trans. Geosci. Remote Sens., vol. 39, no. 5, pp. 1049-1060, May 2001.

[2] A. Camps, M. Vall-llossera, N. Duffo, M. Zapata, I. Corbella, F. Torres, and V. Barrena, "Sun effects in 2-D aperture synthesis radiometry imaging and their cancelation," IEEE Trans. Geosci. Remote Sens., vol. 42, no. 6, pp. 1161-1167, Jun. 2004. 
[3] B. Picard, N. Reul, P. Waldteufel, and E. Anterrieu, "Impact of solar radiation on sea surface salinity remote sensing by spaceborne synthetic aperture imaging radiometers," in Proc. IEEE Int. IGARSS, Sep. 20-24, 2004, vol. 3, pp. 1926-1929.

[4] A. Camps, M. Vall-llossera, N. Reul, F. Torres, N. Duffo, and I. Corbella, "Impact and compensation of diffuse sun scattering in 2D aperture synthesis radiometers imagery," in Proc. IEEE Int. IGARSS, Jul. 25-29, 2005, vol. 7, pp. 4906-4909.

[5] C. T. Swift, "Microwave radiometer measurements of the Cape Cod Cannal," Radio Sci., vol. 9, no. 7, pp. 641-653, 1974.

[6] F. Wentz, "The forward scattering of microwave solar radiation from a water surface," Radio Sci., vol. 13, no. 1, pp. 131-138, 1978.

[7] A. G. Voronovich, "Small-slope approximation in wave scattering by rough surfaces," Sov. Phys._JETP, vol. 62, no. 1, pp. 65-70, 1985.

[8] A. G. Voronovich, "Small-slope approximation for electromagnetic wave scattering at a rough interface of two dielectric half-spaces," Waves Random Media, vol. 4, no. 3, pp. 337-367, Jul. 1994.

[9] P. Beckmann and A. Spizzichino, The Scattering of Electromagnetic Waves From Rough Surfaces. Oxford, U.K.: Pergamon, 1963.

[10] G. A. Duck and D. E. Gary, "The sun at 1.4 GHz," Astron. Astrophys., vol. 124, no. 1, pp. 103-107, 1983.

[11] J. Aarons, Solar System Radio Astronomy. New York: Plenum, 1965.

[12] K. Mursula and T. Ulich, "A new method to determine the solar cycle length," Geophys. Res. Lett., vol. 25, no. 11, pp. 1837-1840, 1998.

[13] K. F. Tapping and D. P. Charrois, "Limits to the accuracy of the $10.7 \mathrm{~cm}$ flux," Sol. Phys., vol. 105, no. 1/2, pp. 305-315, Mar. 1994.

[14] A. G. Voronovich and V. U. Zavorotny, "Theoretical model for scattering of radar signals in $\mathrm{Ku}$ and C-bands from a rough sea surface with breaking waves," Waves Random Media, vol. 11, no. 3, pp. 247-269, Jul. 2001.

[15] T. Elfouhaily, S. Guignard, R. Awadallahand, and D. R. Thompson, "Local and non-local curvature approximation: A new asymptotic theory for wave scattering," Waves Random Media, vol. 13, no. 4, pp. 321-337, 2001.

[16] L. A. Klein and C. T. Swift, "An improved model of the dielectric constant of sea water at microwave frequencies," IEEE Trans. Antennas Propag., vol. AP-25, no. 1, pp. 104-111, Jan. 1977.

[17] V. N. Kudryavtsev, V. K. Makin, and B. Chapron, "Coupled sea surface: Atmosphere model-Part 2: Spectrum of short wind waves," J. Geophys. Res., vol. 104, no. C4, pp. 7625-7639, 1999.

[18] C. Cox and W. Munk, "Statistics of the sea surface derived from sun glitter," J. Mar. Res., vol. 13, no. 2, pp. 198-227, 1954.

[19] C. Cox and W. Munk, Slopes of the Sea Surface Deduced From Photographs of Sun Glitter. Berkeley and Los Angeles, CA: Univ. California Press, 1956, pp. 401-488.

[20] V. Kudryavtsev, D. Hauser, G. Caudal, and B. Chapron, "A semi-empirical model of the normalized radar cross-section of the sea surface-Part 1: Background model," J. Geophys. Res., vol. 108, no. C3, p. 8054, 2003. DOI: 10.1029/2001JCOO1003.

[21] A. Mouche, D. Hauser, and V. Kudryavtsev, "Radar scattering of the ocean surface and sea-roughness properties: A combined analysis from dual-polarizations airborne radar observations and models in C band," J. Geophys. Res., vol. 111, no. C9, p. C09004, 2006. DOI:10.1029/ 2005JC003166.

[22] A. Camps, J. Bar, I. Corbella, and F. Torres, "The processing of hexagonally sampled signals with standard rectangular techniques: Application to 2-D large aperture synthesis interferometric radiometers," IEEE Trans. Geosci. Remote Sens., vol. 35, no. 1, pp. 183-190, Jan. 1997.

[23] D. R. Thompson, T. M. Elfouhaily, and J. Garrison, "An improved geometrical optics model for bistatic GPS scattering from the ocean surface," IEEE Trans. Geosci. Remote Sens., vol. 43, no. 12, pp. 2810-2821, Dec. 2005.

[24] O. Germain, G. Ruffini, F. Soulat, M. Caparrini, B. Chapron, and P. Silvestrin, "The Eddy Experiment: GNSS-R speculometry for directional sea-roughness retrieval from low altitude aircraft," Geophys. Res. Lett., vol. 31, p. L21 307, 2004. DOI:10.1029/2004GL020991.

[25] C. Bourlier and G. Berginc, "Microwave analytical backscattering models from randomly rough anisotropic sea surface-Comparison with experimental data in C and Ku bands," Prog. Electromagn. Res., vol. 37, pp. 31-78, 2002. DOI:10.2528/PIER0110080a.

[26] T. Elfouhaily, B. Chapron, K. Katsaros, and D. Vandemark, "A unified directional spectrum for long and short wind-driven waves," J. Geophys. Res., vol. 102, no. C7, pp. 15781-15796, Jul. 1997.

[27] G. B. Arfken and H. J. Weber, "Mathematical methods for physicists 6th ed.," in Materials and Manufacturing Processes. New York: Academic, 2005.

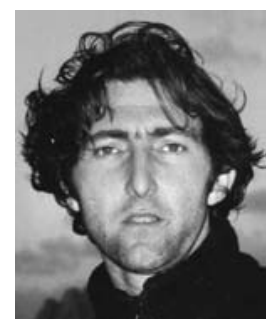

Nicolas Reul received the B.S. degree in marine science engineering from Toulon University, Toulon, France, in 1993 and the Ph.D. degree in physics (fluid mechanics) from the University of Aix-Marseille II, Marseille, France, in 1998

From 1999 to 2001, he was a Postdoctoral Researcher in the team of Prof. M. Donelan with the Department of Applied Marine Physics, Rosenstiel School of Marine and Atmospheric Science, University of Miami, Miami, FL. Since 2001, he has been a Research Scientist with the Spatial Oceanography group, Laboratoire d'Océanographie Spatiale, Institut Français de Recherche et d'Exploitation de la Mer, Plouzané, France, where he is responsible for the activities concerning the future Soil Moisture and Ocean Salinity (SMOS) satellite mission. The focus of his research program is to improve understanding of the physical processes at the air-sea interface and passive/active remote sensing of the ocean surface. He has experience in applied mathematics, physical oceanography, and electromagnetic wave theory and its application to ocean remote sensing.

Dr. Reul is a member of the European Space Agency/SMOS Science Advisory Group.

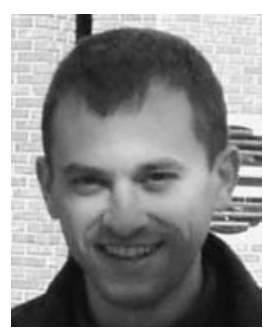

Joseph Tenerelli was born in 1970. He received the B.S. degree in atmospheric sciences from the University of Washington, Seattle, in 1994.

From 1999 to 2005, he was a Research Associate with the Rosenstiel School of Marine and Atmospheric Science, University of Miami, Miami, FL, where he was part of a team that developed a coupled atmosphere-ocean-surface-wave version of an atmospheric mesoscale model (MM5) with vortex-following mesh refinement suitable for simulating hurricanes. Since April 2005, he has been a Research Engineer with the Laboratoire d'Océanographie Spatiale, Institut Français de Recherche et d'Exploitation de la Mer, Plouzané, France, as part of a team developing an algorithm to retrieve sea surface salinity from L-band radiometric measurements (the European Space Agency's SMOS project).

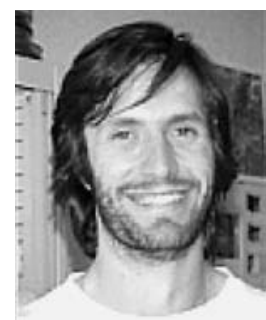

Bertrand Chapron received the B.Eng. degree from the Institut National Polytechnique de Grenoble, Grenoble, France, in 1984 and the Ph.D. degree in physics (fluid mechanics) from the University of Aix-Marseille II, Marseille, France, in 1988.

He spent three years as a Postdoctoral Research Associate at the National Aeronautics and Space Administration (NASA)/Goddard Space Flight Center Wallops Flight Facility, Wallops Island, VA. He is a Research Scientist and is currently the Head of the Spatial Oceanography group at the Laboratoire d'Océanographie Spatiale, Institut Français de Recherche et d'Exploitation de la Mer, Plouzané, France, where he is responsible for the Centre ERS Archivage et Traitement. He was a Coinvestigator and Principal Investigator in several European Space Agency (e.g., ENVISAT, Global Navigation Satellite System), NASA, and CNES (TOPEX/POSEIDON, JASON) projects. He was also coresponsible (with H. Johnsen of NORUT) for the ENVISAT ASAR-wave mode algorithms and scientific preparation for the ENVISAT wind and wave products. He has experience in applied mathematics, physical oceanography, and electromagnetic wave theory and its application to ocean remote sensing.

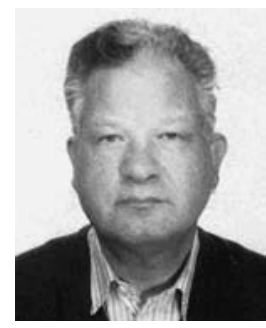

Philippe Waldteufel was born in 1941. He received the B.Eng. degree from the Ecole Polytechnique, Paris, France, in 1962 and the Doctorat d'Etat degree from the Universite de Paris, Paris, in 1970.

$\mathrm{He}$ is currently a Senior Scientist with the Institut Pierre et Simon Laplace, Service d'Aéronomie, Centre National de la Recherche Scientifique, Paris. He has also served as the Director of the Institut de Physique du Globe de Clermont-Ferrand, the Deputy Director of the French Weather Service Research Department, the Director for Science of the French Research Ministry, and the Head of Service d'Aéronomie. His scientific interests include ionospheric and thermospheric physics, radar meteorology, radiowave propagation, macroeconomics, and microwave radiometry. 